\title{
The evolutionary history of the polyQ tract in huntingtin sheds light on its functional pro-neural activities
}

\author{
Raffaele lennaco ${ }^{1,2,8}$, Giulio Formenti ${ }^{1,2,8}$, Camilla Trovesi ${ }^{1,2,8}$, Riccardo Lorenzo Rossi ${ }^{2,8}$, Chiara Zuccato ${ }^{1,2,8}$, Tiziana Lischetti ${ }^{1,2,8}$, \\ Vittoria Dickinson Bocchi ${ }^{1,2}$, Andrea Scolz ${ }^{1,2}$, Cristina Martínez-Labarga ${ }^{3}$, Olga Rickards ${ }^{3}$, Michela Pacifico ${ }^{1,2}$, Angelica Crottini ${ }^{4}$, \\ Anders Pape Møller ${ }^{5}$, Richard Zhenghuan Chen ${ }^{6}$, Thomas Francis Vogt ${ }^{6}$, Giulio Pavesi ${ }^{1}$, David Stephen Horner ${ }^{1}$, Nicola Saino ${ }^{7,9}$ and \\ Elena Cattaneo (D) $^{1,2 \text { 屾 }}$
}

(c) The Author(s) 2021

Huntington's disease is caused by a pathologically long (>35) CAG repeat located in the first exon of the Huntingtin gene (HTT). While pathologically expanded CAG repeats are the focus of extensive investigations, non-pathogenic CAG tracts in protein-coding genes are less well characterized. Here, we investigated the function and evolution of the physiological CAG tract in the HTT gene. We show that the poly-glutamine (polyQ) tract encoded by CAGs in the huntingtin protein (HTT) is under purifying selection and subjected to stronger selective pressures than CAG-encoded polyQ tracts in other proteins. For natural selection to operate, the polyQ must perform a function. By combining genome-edited mouse embryonic stem cells and cell assays, we show that small variations in HTT polyQ lengths significantly correlate with cells' neurogenic potential and with changes in the gene transcription network governing neuronal function. We conclude that during evolution natural selection promotes the conservation and purity of the CAG-encoded polyQ tract and that small increases in its physiological length influence neural functions of HTT. We propose that these changes in $\mathrm{HTT}$ polyQ length contribute to evolutionary fitness including potentially to the development of a more complex nervous system.

Cell Death \& Differentiation (2022) 29:293-305; https://doi.org/10.1038/s41418-021-00914-9

\section{INTRODUCTION}

Huntington's disease (HD) is a neurological genetic disorder caused by an anomalous expansion of a $>35$ CAG triplet repeats in the huntingtin gene $(H T T)$ that leads to an abnormally elongated polyglutamine (polyQ) tract [1-3]. It is currently unknown whether HD pathogenesis occurs at the DNA, RNA, or protein levels [4-7]. Within the non-pathogenic range of CAGs, the HTT gene encodes for a protein (HTT) which is implicated in multiple fundamental physiological processes. Among these functions, HTT regulates neurogenesis and brain development, together with the survival and function of cortical and striatal neurons in the adult brain $[8,9]$. Molecularly, HTT scaffolds a large repertoire of binding proteins to coordinate several cellular processes, including gene transcription, synaptic activity, vesicles trafficking and autophagy [8-10]. In spite of this knowledge, the contribution of the nonpathogenic CAG tract itself and its highly polymorphic lengths in normal $H T T$ function remains poorly examined.

In the general human population, the non-pathogenic CAGs number ranges from 9 to 35 . More remarkably, its distribution is right-skewed [11-13] with a higher frequency of longer CAG tracts in the highest normal CAGs range between 27-35 [12], thus suggesting that in humans there is a bias towards longer alleles transmission. This unexpected finding, together with the evidence that CAGs have arisen and have been retained throughout evolution suggest that CAG encoding repeats may have a positive role in adaptive evolution and might exert a function [14-19].

Earlier hypothesis proposed that the CAG repeat tracts have little or no effect on the normal function of the gene in which they are located [20,21]. In contrast, initial studies in humans reveal that longer non-pathogenic CAG size in HTT gene are associated with a phenotype. In particular, CAG repeats below disease threshold were described to have an impact on brain development and general intelligence [22]. Furthermore, the size of the non-pathogenic trinucleotide repeats has been found to affect normal brain structure in humans [23], and more CAGs in the nonpathogenic range were suggested to have an impact on cognition $[24,25]$. Together, these data indicate that the non-pathogenic CAG tract in HTT may confer advantageous effects to the DNA, RNA and/or protein. Moreover, our earlier work speculated that CAGs may have been subjected to selective pressure during evolution because greater number of repeats are found in species sharing a more recent common ancestor with humans. We

\footnotetext{
${ }^{1}$ Department of Biosciences, University of Milan, 20122 Milan, Italy. ${ }^{2}$ Istituto Nazionale Genetica Molecolare, Romeo ed Enrica Invernizzi, 20122 Milan, Italy. ${ }^{3}$ Department of Biology, University of Roma Tor Vergata, 00173 Rome, Italy. ${ }^{4} \mathrm{CIBIO}$, Research Centre in Biodiversity and Genetic Resources, InBIO, Universidade do Porto, 4485-661 Vairão, Portugal. ${ }^{5}$ Ecologie Systématique Evolution, Université Paris-Sud, CNRS, Agro ParisTech, Université Paris-Saclay, 91405 Orsay, CEDEX, France. ${ }^{6} \mathrm{CHDI}$ Management/CHDI

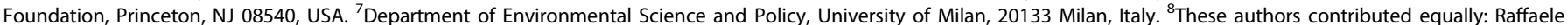
lennaco, Giulio Formenti, Camilla Trovesi, Riccardo Lorenzo Rossi, Chiara Zuccato, Tiziana Lischetti. ${ }^{9}$ Deceased: Nicola Saino. ${ }^{凶}$ email: elena.cattaneo@unimi.it Edited by G. Melino
}

Received: 24 June 2021 Revised: 9 November 2021 Accepted: 24 November 2021

Published online: 1 January 2022 
reported that Echinodermata and Cephalochordata are the first groups of organisms to carry a polyQ (only 2Q) tract in the HTT protein $[17,26]$, and curiously are among the most basal lineages to develop a primitive nervous system [27, 28]. Vertebrates, instead, have four or more Qs, with the highest number found in humans [17]. These results again support the hypothesis that the CAG sequence, which has survived and has been preserved to this day, has a fundamental role and a defined purpose.

Here we directly tested the hypothesis that the length and purity of the non-pathogenic CAG tract in $H T T$ have been promoted by natural selection and that the tract length is functionally active. First, by studying the conservation of the CAG tract from over 200 species, the largest sequencing effort of $H T T$ exon1 to date, we provide evidence that the HTT polyQ tract is under purifying selection and subjected to stronger selective pressures than any other polyQ-containing protein. Second, by using genome-edited mouse embryonic stem (mES) cells that carry a stepwise increase of CAGs in the normal HTT gene, we show that the polyQ tract itself is functionally relevant. Importantly, small increases in the non-pathogenic CAG length positively and significantly correlate with the cells' neurogenic potential and with transcriptional alterations in pathways that control general neuronal organization and function/s. Taken together, these results demonstrate that natural selection acts to preserve an uninterrupted contiguous $\mathrm{Q}$ stretch in $\mathrm{HTT}$ and that its progressive increase in length gradually enhances the biological, pro-neurogenic activity of the normal protein as we demonstrate by stem cell based assays.

\section{MATERIALS AND METHODS \\ Sequence retrieval from databases}

Sequences were obtained from four online resources: NCBI (www.ncbi.nlm. nih.gov), UCSC (www.genome.ucsc.edu), Ensembl (www.ensembl.org) and UNIPROT (www.uniprot.org). Coding sequences were obtained as nucleotide mRNA predictions either directly or as GenBank files. BLAST searches using Human $H T T(\mathrm{Hg} 18)$ full-length exon1 sequence were performed in unannotated genomic assemblies. Specific SRA (Sequence Read Archive) experiments were also searched for $H T T$ orthologous genes using sequences from relatively close species as queries.

\section{Sample collection and extraction of genomic DNA}

gDNA extraction was performed either using a slightly modified Phenol/ Chloroform protocol or using a commercial kit (NucleoSpin Tissue ${ }^{\circledR}$ kitMachery-Nagel, REF 740952). Quality and concentration of all extracted DNA samples were verified on a gel and by spectrometer analysis (NanoDrop 1000-ThermoScientific).

\section{Amplification of HTT exon 1 orthologs}

A general PCR protocol was developed to assess the nucleotide sequence of exon 1 in the HTT orthologous genes. Primers were designed in the most conserved outer regions of $H T T$ exon 1 to span the entire repeat region and to produce amplicons between $100-300 \mathrm{bp}$, suitable for direct sequencing or cloning (see Supplementary dataset S22 for the primer list). A topquality replication enzyme, the Platinum Taq DNA Polymerase High Fidelity (kit Life Technologies, CAT $n^{\circ} 11304-011$ ) was used for PCR amplification. PCR reactions were assembled under a sterile fume hood and working environment was cleaned with a nucleic acids decontamination solution (NucleoClean ${ }^{\mathrm{TM}}$ Decontamination Solution, Merck Millipore $n^{\circ}$ 3097S) prior to mix preparation. A general PCR protocol with slight modifications was applied on all vertebrate gDNA (see Supplementary dataset S22). PCR products quality and length were assessed by gel electrophoresis and negative and positive controls were always included. Whenever possible, more than one sample per species has been tested (as listed in Supplementary dataset S1).

\section{Amplification of pHTT}

The $p H T T$ belonging to Callithrix jacchus was amplified according to the genome information (Ensembl release 91, C_jacchus3.2.1). The reaction was performed in a total volume of $15 \mu \mathrm{l}$ with $1 \mu \mathrm{l}$ of genomic DNA (buffer
HF $1 \times, \mathrm{MgSO}_{4} 0.8 \mathrm{mM}$, dNTPs $0.2 \mathrm{mM}$, Primer FW $0.35 \mu \mathrm{M}$, Primer RV $0.35 \mu \mathrm{M}$, Taq Platinum HF $5 \mathrm{U} / \mu \mathrm{l}$, DNA $\sim 100 \mathrm{ng} / \mu \mathrm{l}$; Primer FW: $5^{\prime}-\mathrm{GC}$ GACCCTGGAAAAGCTGAT-3'; Primer RW: 5'-TGGTCAGGGCTTGCAGAAG-3'). The amplification consisted of $10^{\prime}$ at $96^{\circ} \mathrm{C}$; 36 cycles of $45^{\prime \prime}$ at $96^{\circ} \mathrm{C}, 30^{\prime \prime}$ at $58^{\circ} \mathrm{C}, 1^{\prime} 30^{\prime \prime}$ at $68^{\circ} \mathrm{C} ; 10^{\prime}$ at $68^{\circ} \mathrm{C}$. PCR products quality and length $(\sim 1100$ bp) were assessed by gel electrophoresis. PCR products were sequenced, using Sanger technology, either directly or after cloning.

\section{Cloning and sequencing of PCR products}

TOPO TA Cloning Kit (Life Technologies, CAT ${ }^{\circ}$ K4575) was used to clone the PCR amplification product for direct sequencing. A commercial kit (Qiagen, CAT $n^{\circ}$ 27106) was used to perform the DNA mini-preparation. $P C R$ product purification and sequencing reactions were carried out on $A B I$ machines according to manufacturer specification either by us or MWG Operon (Eurofins Genomics, Ebersberg, Germany) and GATC Biotech AG (Costance, Germany). PCR products were Sanger sequenced either directly or after cloning. Technical replicates of sequencing were performed. More than one sample per species, when available, has been sequenced (as listed in Supplementary dataset S1).

\section{Alignment of exon 1 orthologs}

Multiple sequence alignments (MSA) were conducted with Clustal Omega [29]. Alignment refinements were performed manually in SeaView 4 (http://doua.prabi.fr/software/seaview), CLC Sequence Viewer (www. qiagenbioinformatics.com) and Geneious version 9 (www.geneious.com) (the overall alignment used is available as Supplementary dataset S2, as well as taxa subgroups alignments are available as supplementary nexus files).

\section{Test for purifying selection}

The tests for negative/positive selection were performed on MSA organized as Nexus files with custom evolutionary trees. For all analyses, MSAs including gap (e. g. CCN region in mammals) and unknown regions (i.e., primer bind and LQ base calling) were employed. Phylogenetic tree topologies for sampled organisms were obtained from the TimeTree.org resource (http://www.timetree.org), exported in the Newick format and embedded in the Nexus files containing the MSAs. To estimate the extent of pervasive purifying selection at individual sites we used three different methods: the Single-Likelihood Ancestor Counting (SLAC) method [30], the Fixed Effects Likelihood (FEL) method [30], and the Fast Unconstrained Bayesian AppRoximation (FUBAR) method [31]. SLAC is the simplest inference legacy method: it is based on counting how many synonymous and non-synonymous changes have occurred at each site over evolutionary time and uses the binomial distribution as a statistical test [30]. FEL instead estimates a value for $\mathrm{dN}$ and $\mathrm{dS}$ at each considered site testing it using a maximum likelihood approach and a chi squared distribution [30]. FUBAR is particularly efficient on larger datasets (high number of sequences) and takes a Bayesian approach to selection inference developed in the context of document classification (latent Dirichlet allocation) [31]. Finally, FUBAR also calculates an a priori probability over a specified grid of $d N$ and $d S$ values thus defining a space of probability over a wide range of selection scenarios.

Calculations were performed using implementations on the Datamonkey Servers (version 2, www.datamonkey.org/) and/or a local instance of the HyPhy software (version 2.2.4), and all methods were used with built-in defaults parameters. The taxa list of the species initially considered and the Nexus input files used for the purifying selection analyses after pruning (HyPhy discards identical sequences) are available as supplementary data files.

\section{Retrieval and statistical testing of phylogenetically conserved polyCAG/CAA genes}

All human (genome assembly hg19) coding sequence (CDS) transcripts with at least four consecutive glutamines were downloaded from Ensembl and for each of these genes the longest CDS was considered. Sequence orthologs were retrieved from Ensembl for all available mammalian genomes and aligned at the protein level using Clustal Omega [29]. Codon alignments were reconstructed using custom Python scripts. For each nonhuman sequence, the region of the alignment corresponding to the human polyQ tract was examined and the longest pure glutamine stretch overlapping the human stretch or conjoined alignment gaps was considered. Subsequently, the longest Q stretch (LQ) and the longest non-interrupted CAG interval (LNI) was calculated for each of these 
stretches. To reduce the impact of phylogenetic structure, only taxa that had a curated $H T T$ sequence were considered. Conserved tracts were selected based on their presence in more than five primate and five nonprimate genomes for which $\mathrm{HTT}$ sequences were available (considering those species for which the $\mathrm{HTT}$ sequence was also available). Only polyQ tracts that displayed at least two different polyCAG/CAA lengths were tested.

A standard score (z-score)-like statistic was calculated for $\mathrm{LQ}, \mathrm{LNI}$, and $\mathrm{PQ}$ (purity of the polyQ tract, i.e., CAG/CAA proportion) for each polyQ tract [e.g., (LQ-mean LQ for orthologous tracts)/standard deviation of LQ for orthologous tracts], and the frequency distributions of these scores were considered.

\section{HTT and pHTT comparative analysis}

General Time Reversible (GTR) model (with four discrete gamma distributed substitution rate categories) was used to estimate numbers of substitutions in Callithrix jacchus HTT and PHTT sequences under maximum likelihood criterion (using PAUP [32]) with Aotus nancymaae HTT sequence as outgroup. All rate parameters were estimated on the alignment and tree topology. The rates of substitutions are represented as the ratio between observed and expected substitutions, according to the length of each exon. Expected values were calculated on the basis of the total number of substitutions and the total length of the alignment, and scaled to the exon lengths.

\section{Mouse embryonic stem ( $m E S)$ cell lines}

We used mouse E14 ES cell lines [33]: parental $(+/+)$, heterozygous $(+/-)$, or homozygous (-I-; RMCE/-) knock-out for the HTT gene. RMCE/- cell line was used to generate $H T T$ knock-in cell lines $(0 \mathrm{Q}, 2 \mathrm{Q}, 4 \mathrm{Q}, 7 \mathrm{Q}, 10 \mathrm{Q}, 13 \mathrm{Q}$, and 17Q). Q banding karyotype analysis performed by Integrated Systems Engineering srl (ISENET) on $\pm /+,-/-$, and RMCE/- cell lines confirmed normal karyotype. The expression of OCT4 and SOX2 pluripotency markers was confirmed by immunofluorescence in all the cell lines generated. We also used R1 mES cell lines [34] expressing the wild-type $H \pi T$ gene $\left(\mathrm{Hdh}^{+/+}\right)$ or being homozygous knock-out for the gene $\left(H d h^{\text {ex4-5 }}\right)$, in which both alleles of the HTT gene were inactivated by deletion of exons 4 and 5 [35]. All transgenic cells were derived by the transfection of pCAG constructs in $H d h^{\text {ex4-5 }}$ cells. Both $\mathrm{Hdh}^{+/+}$and $H d h^{\text {ex4-5}}$ were analysed for karyotypic abnormalities (Q-banding) and they showed several common [36] chromosomal alterations at the cell population level (the most frequent were duplications of chromosomes 1, 8, 11, and 14).

\section{Mouse ES cell culture}

ES cells were maintained in Glasgow minimal essential medium supplemented with $10 \%$ heat-inactivated fetal bovine serum (vol/vol, EuroClone, REF ECS0186L), 0.1 mM $\beta$-mercaptoethanol (Gibco, REF \#31350-010), $100 \mu \mathrm{M}$ non-essential amino acids (Gibco, REF \#11140-035), $1 \mathrm{mM}$ sodium pyruvate (Gibco, REF \#11360-039), 2 mM L-glutamine, $100 \mathrm{U} / \mathrm{ml}$ penicillin, $100 \mu \mathrm{g} / \mathrm{ml}$ streptomycin (EuroClone, REF ECB3001D) and $1000 \mathrm{U} / \mathrm{ml}$ murine leukemia inhibitor factor (LIF, ESGRO) (Millipore, REF ESG1107) in gelatinized tissue culture flasks. Cells were passaged every 2 days after dissociation with $0.05 \%$ trypsin-EDTA (vol/vol) (Gibco, REF 15400-054).

\section{HTT knock-out}

gRNA2p and gRNA5i (supplementary dataset S23), respectively annealing 250 bp upstream and 430 bp downstream $H T T$ exon1, were used to drive its precise deletion in $\mathrm{E} 14+/+\mathrm{mES}$ cells [37]. Alt- $\mathrm{R}^{\otimes}$ CRISPR-Cas9 tracrRNA (IDT) and the two designed Alt-R ${ }^{\circledast}$ CRISPR-Cas9 crRNAs were separately assembled in vitro and added to the Alt- $R^{\circledast}$ S.p. Cas9 nuclease (IDT) following manufacturer's instruction. A mix containing $1.8 \mathrm{pmol}$ of each ribonucleoprotein (RNP) complex was introduced by reverse lipofection in 320,000 E14 +/+ mES cells using Lipofectamine ${ }^{\circledR}$ RNAiMAX (Thermo Fisher). Clones were isolated and screened by PCR for the presence of the small expected knock-out band with oligos crHTTexon $1 \mathrm{kofw}$ and crHTTexon1korev (supplementary dataset S23). Three primers-based PCR assay enabled to better discriminate between homozygous and heterozygous clones (Fig. S7A, B and Supplementary dataset S23). Two validated clones for each genotype were exposed to neural rosette assay.

\section{HTT knock-in}

Two gRNAs cutting $1.5 \mathrm{~kb}$ upstream (Cas9 HAL) and $2 \mathrm{~kb}$ downstream (Cas9 HAR) HTT exon1 were used to integrate the RMCE cassette by homologous-directed recombination (HDR) (supplementary dataset S23). Two million E14 $+/+$ mES cells were nucleofected using Amaxa ${ }^{\mathrm{TM}}$ mES nucleofector kit (Lonza) with a mix containing 20 pmol of each in vitro assembled RNP complex, $10 \mu \mathrm{l}$ nucleofector enhancer (IDT) and $1 \mu \mathrm{g}$ RMCE-Htt-PuroTK donor cassette (synthetized by GenScript and cloned in pUC57). The HDR donor vector contained $1 \mathrm{~kb}$ homology arms and the RMCE cassette, that comprises the FRT and F3 sites specific for the Flp recombinase and the positive/negative selectable marker PuroRATK under the human PGK promoter (supplementary dataset S23). Cells were seeded 35,000 cells $/ \mathrm{cm}^{2}$ and grown in presence of $1 \mu \mathrm{M}$ SCR7 ligase IV inhibitor for $24 \mathrm{~h}$ after nucleofection to promote repair by HDR. Clones resistant to $2 \mu \mathrm{g} / \mathrm{ml}$ puromycin were isolated and screened by PCR for the integration of the RMCE cassette without random insertion of the donor vector at first and subsequently for genotyping the second allele (primer pairs used listed in supplementary dataset S23).

We selected one RMCE/- clone in which we integrated by Flp-mediated recombination $0 \mathrm{Q}, 2 \mathrm{Q}, 4 \mathrm{Q}, 7 \mathrm{Q}, 10 \mathrm{Q}, 13 \mathrm{Q}$, and $17 \mathrm{Q} \mathrm{RMCE}$ constructs (synthetized by GenScript and cloned in pUC57 vector) to reconstitute the whole genomic portion removed by the PuroRATK RMCE cassette. To this aim, 2 million RMCE/- cells were nucleofected using Amaxa ${ }^{\text {TM }} \mathrm{mES}$ nucleofector kit (Lonza) with $4 \mu \mathrm{g}$ RMCE donor construct (either $0 \mathrm{Q}, 2 \mathrm{Q}$, $4 \mathrm{Q}, 7 \mathrm{Q}, 10 \mathrm{Q}, 13 \mathrm{Q}$, or 17Q) and $4 \mu \mathrm{g}$ pCAG-Flpe:GFP (Addgene, plasmid \#13788). Cells were seeded at low density (4-8000 cells $/ \mathrm{cm}^{2}$ ) and exposed to $1 \mu \mathrm{M}$ ganciclovir selection $72 \mathrm{~h}$ after nucleofection. Recombination efficiency resulted to be higher than $60 \%$ for all constructs. Clones were individually validated and two clones for each genotype were exposed to neural rosette assay.

\section{Clone validation}

Sequencing of HTT locus. PCR products from HTT locus were purified using QIAquick PCR-purification Kit (QIAGEN) and Sanger sequenced (GATCEurofins).

$H T T$ expression. RNA was isolated from clones of interest with TRIzol reagent according to the manufacture's instruction (Life Technologies) and reverse transcribed with iScript CDNA Synthesis Kit (Bio-Rad). The presence of HTT mRNA was evaluated using primers amplifying exon 3-6 CDNA (supplementary dataset S23) and HTT expression was checked by western blot analysis using D7F7 antibodies (Cell Signaling Technology).

\section{Transgenic mouse ES cell lines}

Constructs. The N-terminal portions of mouse $(\mathrm{Mm}) \mathrm{HTT}$ were designed according to previously published HTT MSA [17] and cloned in PCAG constructs: pCAG Mm 0Q, pCAG Mm 2Q, pCAG Mm 4Q, pCAG Mm 7Q, pCAG $M m$ Q3PQ3, pCAG $M m$ Q3Q(CAA)Q3.

DNA transfections. Hdh ${ }^{\text {ex4-5 }}$ ES cells were plated at a density of $2 \times 10^{4}$ cells per $\mathrm{cm}^{2}$. After $24 \mathrm{~h}$, cells were transfected with different expression vectors $(10 \mu \mathrm{g})$ and Lipofectamine 2000 (Thermo Fisher Scientific, REF $11668027)$. Cells were selected with puromycin $\left(2 \mu \mathrm{m} \mathrm{m}^{-1}\right) 24 \mathrm{~h}$ after lipofection for 10 days. Western blot and immunofluorescence were performed to determine the expression of the transgene.

\section{Protein lysates and western blots}

Cells were lysed in RIPA buffer ( $50 \mathrm{mM}$ Tris- $\mathrm{HCl}$ pH $8,150 \mathrm{mM} \mathrm{NaCl}, 0.1 \%$ SDS, $1 \%$ nonidet P40, $0.5 \%$ sodium deoxycholate, wt/vol) with $1 \mathrm{mM}$ PMSF and protease inhibitor (Thermo Scientific, REF 1861281). Lysates were cleared by centrifugation at $12,000 \times \mathrm{g}$ and $4^{\circ} \mathrm{C}$ for $30 \mathrm{~min}$. The resulting supernatant was collected. Protein concentration was determined with the Pierce-BCA Protein assay kit (Thermo Scientific REF 23225) and $30 \mu \mathrm{g}$ were loaded on a 7.5\% SDS-PAGE gel. Separated proteins were transferred to a nitrocellulose membrane, blocked with $5 \%$ non-fat dry milk (wt/vol, Biorad, REF 170-6404) in Tris-buffered saline (TBS) and 0.1\% Tween-20 ( $\mathrm{vol} / \mathrm{vol})$, and incubated with primary antibody at room temperature $\left(20-23^{\circ} \mathrm{C}\right)$ for 3 h. After washing, filters were incubated for $1 \mathrm{~h}$ at room temperature with a secondary antibody (peroxidase conjugate, Biorad, 1:3000) and then washed three times with TBS and $0.1 \%$ Tween-20. The Clarity Western ECL Substrate (Biorad, REF 170-5061) was used to visualize immunoreactive bands by chemiluminescence detection with ChemiDoc MP Imaging System (Biorad).

\section{Mouse ES cell monolayer differentiation}

ES cells were dissociated and plated onto $0.1 \%$ gelatin-coated tissue culture dishes at a density of $1.6-2.4 \times 10^{4}$ cells per $\mathrm{cm}^{2}$ in N2B27 medium. N2B27 medium was a 1:1 mixture of DMEM/F12 and Neurobasal medium 
containing 1:200 N2 supplement (Gibco, REF 17502-048), 1:100 B27 supplement (Gibco, REF 17504-044) and $0.1 \mathrm{mM} \beta$-mercaptoethanol (Gibco, REF 31350-010). Medium was renewed every 2 days. Neural rosettes formed by progenitor cells were analysed after 8-9 days of differentiation

For the terminal differentiation process, on day 12-14 neural progenitors were dissociated with Accutase (Millipore, REF SF006) and plated onto $10 \mathrm{ng} / \mu$ l Poly-D-Lysin (Sigma Aldrich, REF P6407) and $3 \mathrm{ng} / \mu \mathrm{l}$ Lamin (Thermo Fisher, REF 23017015) coated tissue culture dishes at density of $1 \times 10^{5}$ cells per $\mathrm{cm}^{2}$ in N2B27 post re-plating medium. N2B27 post re-plating medium was a 1:4 mixture of DMEM/F12 and Neurobasal medium containing 1:100 N2 supplement (Gibco, REF 17502-048), 1:50 B27 supplement (Gibco, REF $17504-044$ ), and $30 \mathrm{ng} / \mathrm{ml}$ Brain-derived neurotrophic factor (PeproTech, REF 450-02). Medium was renewed every 2 days. Terminal differentiated cell cultures were analysed after 28 days of differentiation.

\section{Immunofluorescence}

Cells were fixed in $4 \%$ paraformaldehyde for $15 \mathrm{~min}$ at $20-23^{\circ} \mathrm{C}$. Cells were permeabilized and blocked in blocking buffer containing PBS with $0.5 \%$ Triton X-100 (vol/vol) and with $5 \%$ fetal bovine serum (vol/vol) for $1 \mathrm{~h}$. Primary antibodies were diluted in blocking buffer and applied overnight at $4{ }^{\circ} \mathrm{C}$. After three washes in PBS, appropriate secondary antibodies, conjugated to Alexa fluorophores 488 or 568 (Molecular Probes, Invitrogen) and diluted 1:500 in blocking solution, were applied for $1 \mathrm{~h}$ at room temperature $\left(20-23^{\circ} \mathrm{C}\right)$. Cells were incubated for $10 \mathrm{~min}$ with Hoechst $33258(5 \mu \mathrm{g} / \mathrm{ml}$, Molecular Probes, Invitrogen). Images were acquired with a Leica DMI 6000B microscope (equipped with LAS-AF imaging software) or with an IN Cell Analyzer 6000 (GE Healthcare Life Sciences) and processed with the software ImageJ (US National Institutes of Health) and CellProfiler (vers.2.1.1).

\section{Rosette and neuron quantification}

Rosette quantification. In the E14 cell differentiation experiments, the rosette lumen areas were quantified on day 8-9 of neural differentiation after staining for NESTIN and PALS1. CellProfiler software was used to automatically quantify rosette lumens (PALS1 positive areas). In the R1 cell differentiation experiments, rosettes were quantified on day 7 of neural differentiation after staining for NESTIN and ZO1. For the measurement of the $\%$ of NESTIN+ cells inside rosettes a previously established protocol was applied [18]. For rosette and lumen size, the contour of each rosette (as highlighted by marker NESTIN) and lumen (as highlighted by marker ZO1) was outlined using ImageJ software and two lists of regions of interest (ROIs) were measured to calculate rosette mean areas and lumen mean areas, respectively.

Neuron quantification. In the E14 cell terminal differentiation experiments, the neurons were analysed on day 28 of neural differentiation after staining for MAP2, TUBB3, P27, and NEUN. CellProfiler software was used to automatically quantify MAP2 and TUBB3 positive areas as well as P27 and NEUN positive cells. The relative number of P27 and NEUN positive cells was calculated as a percentage of total Hoechst positive cells.

\section{Antibodies}

The antibodies and dilutions used for immunofluorescence and western blotting were: monoclonal antibody to Huntingtin (Millipore, Mab2166; immunofluorescence, 1:100; western blotting, 1:1000; Cell Signaling Technology, D7F7, western blotting, 1:1000), monoclonal antibody to NESTIN (Millipore, Mab353; immunofluorescence, 1:200), polyclonal antibody to ZO1 (Life Technologies, REF 402300; immunofluorescence, 1:200), polyclonal antibody to PALS1 (Proteintech, 17710-1-AP; immunofluorescence, 1:500), monoclonal antibody to OCT4 (Santa Cruz, sc-5279; immunofluorescence, 1:100), polyclonal antibody to SOX2 (Millipore, AB5603; immunofluorescence, 1:200), polyclonal antibody to TUBB3 (BioLegend, 802001, immunofluorescence, 1:1000), monoclonal antibody to MAP2 (BD, 556320, immunofluorescence, 1:500), monoclonal antibody to P27 (Cell Signaling Technology, 3698, immunofluorescence, 1:1000), monoclonal antibody to NEUN (Millipore, Mab377; immunofluorescence, 1:100).

RNA extraction (for real-time qPCR or RNA-seq analyses)

RNA was isolated using TRlzol reagent according to the manufacture's instruction (Life Technologies). RNA was quantified with Nanodrop and then the integrity was evaluated. Potential contaminating DNA was removed by DNA-free kit (Ambion). RNA was reverse transcribed using iScript cDNA Synthesis Kit (Bio-Rad).

\section{Real-time qPCR}

qPCR was performed using the CFX96 Real-Time System (Bio-Rad). All reactions were performed in $15 \mu \mathrm{l}$ containing $50 \mathrm{ng}$ (or $100 \mathrm{ng}$ for $\mathrm{SHH}$ and PLAGL1) cDNA and SsoFast EVAGreen Supermix (Bio-Rad). Primer pairs used were listed in supplementary dataset S23.

\section{Apoptosis assay}

Cells were seeded in triplicate in two 96-well plates, at $16 \times 10^{3}$ cells per well. After $24 \mathrm{~h}$ incubation at $37^{\circ} \mathrm{C}$, cells received $100 \mu \mathrm{l}$ of freshly prepared GMEM + LIF without serum. At $48 \mathrm{~h}$ after plating, $100 \mu \mathrm{l}$ of CellTiter Reagent (Promega, REF G7570) was added to all wells. After $10 \mathrm{~min}$ of incubation at $20-23^{\circ} \mathrm{C}$, luminescence was measured with a VeritasMicroplateLuminometer (Turner Biosystems) to detect cell viability. Caspase-Glo 3/7 Reagent ( $100 \mu l$, Promega, REF G8091) was added to all parallel wells. After $30 \mathrm{~min}$ of incubation at $20-23^{\circ} \mathrm{C}$, luminescence was recorded with a Veritas-MicroplateLuminometer (Turner Biosystems) to detect CASPASE-3/7 activity. Each experiment was independently repeated at least three times. Results are presented as the ratio of CASPASE-3/7 luminescence/cell viability luminescence recorded for each cell line.

\section{Statistical analyses of biological assays}

Data from the quantification of the rosette, neuron and apoptosis assays were analysed with one-way ANOVA by using GraphPad Prism (version 7). Posthoc comparisons between cell lines were performed by applying Tukey or Bonferroni procedure. Correlation analysis between $Q$ lengths and phenotypes were calculated by linear regression analysis (Pearson's and Spearman's tests).

\section{RNA sequencing and analyses}

Sequencing. RNA-seq was performed on $0 \mathrm{Q}, 2 \mathrm{Q}, 4 \mathrm{Q}$, and $7 \mathrm{Q}$ cell lines (three biological replicates per cell line) at the rosette (day 8) and neuronal (day 29) stage. Stranded polyA libraries were sequenced on the Illumina HiSeq 4000 platform, with an average depth of 40 million $150 \mathrm{bp}$ paired-end reads per sample.

RNA-seq quality control and mapping. The quality of the reads was tested with MultiQC [38] before and after a trimming step performed using Trimmomatic [39]. Low-quality reads or reads shorter that 50 bases were removed. Adapters and low-quality bases (quality $<3$ ) in both leading and trailing ends of the reads as well as any four consecutive bases with an average quality per base less than 15 were also removed. Reads were then aligned to the mouse reference genome (GRCm38.98, with gene annotation GENCODE M25) using STAR v2.5.2b [40] with default parameters.

RNA-seq data processing. Differential expression analysis was performed with DESeq2 [41]. To take into account experimental batches, replicates were added as additional variables and included into the design formula.

At the rosette and neuronal stages all the possible pairwise comparisons were performed $(0 Q$ vs. $2 Q, 0 Q$ vs. $4 Q, 0 Q$ vs. $7 Q, 2 Q$ vs. $4 Q, 2 Q$ vs. $7 Q$, and $4 Q$ vs. $7 Q$ ). Genes were considered differentially expressed if the adjusted $p$-value (Benjamini Hochberg) was lower than 0.05 and the absolute log2 fold change was higher than 1 . Genes with low base mean $[<1$ CPM (counts per million)] were not considered in the analysis.

Functional analysis. Enrichment analysis for Gene Ontology (GO) terms was performed using TopGO [42], focusing on the "Biological Process" category. GO terms with Fisher test corrected $p$-values $<0.05$ were considered as significant. The GOplot package [43] was used to generate circular plot and compute $z$-scores (number of upregulated genes minus the number of downregulated genes divided by the square root of the count).

"Jaccard similarity" index. The analysis was performed by comparing the top 100 Differentially Expressed Genes (DEGs) between rosettes (early stage) and neurons (terminal stage) of $0 \mathrm{Q}, 2 \mathrm{Q}, 4 \mathrm{Q}$, and $7 \mathrm{Q}$ neuronal cultures with the top 100 unique DEGs from bulk RNA-seq data of human fetal cortical (CTX), striatal (Lateral Ganglionic Eminence, LGE) and pallidal (Medial Ganglionic Eminence, MGE) tissues [44].

\section{RESULTS}

\section{The HTT polyQ tract is under purifying natural selection}

We started by investigating whether long-term patterns of conservation and change for HTT CAG repeats have been subjected to selective pressures. To this aim, we assembled a 
Multiple Sequence Alignment (MSA) of HTT exon1 sequences from more than 200 chordate species-108 de novo sequenced orthologs and 101 sequences retrieved from online databases or published studies (Supplementary datasets S1 and S2). Our MSA displays variation in the length of the HTT polyQ tract across chordate groups (Fig. S1). To understand whether selective pressures contributed to these patterns, we measured first the ratio of nonsynonymous to synonymous substitutions ( $d N / d S$ ratio) for each position of this locus. A low rate of between-species nonsynonymous substitution - that is a nucleotide mutation that alters the amino acid sequence of a protein-reflects the action of purifying selection within species to eliminate mildly deleterious mutations and preserve the tract intact. In our analyses we estimated the substitution occurrences for 163 non-redundant (unique) highly confident exon 1 sequences (Supplementary dataset S3). Importantly, we found that only two of the 291 inferred non-synonymous substitutions within the exon 1 locus fall within the polyQ tract (Fig. 1A).

Second, for a more rigorous conservation/selective pressure analysis on the polyQ tract, we quantified the $\mathrm{d} N / \mathrm{d} S$ ratio. When there are substantial variations in CAG repeat length between species-driven by repeat expansion/contraction events-the region could not be reliably aligned because the $N$ th CAG (or Q) in one species could not be orthologous to the Nth CAG repeat in a different species. Being aware of this possible limitation, we assessed $\mathrm{d} N / \mathrm{d} S$ ratio on non-mammalian sequences with stable $4 \mathrm{Q}$ repeats ( $n=84$ species) (Supplementary dataset S4)-as the absence of indels allows confident alignment across their entire length. The most commonly used approaches to assess positive or negative (purifying) selection through the $\mathrm{d} N / \mathrm{d} S$ ratio across the phylogeny are SLAC, FEL [30], and FUBAR [31], which are based on different algorithms and statistical tests (see "Methods" section). All these approaches (FUBAR, Fig. 1B; SLAC and FEL, Fig. S2A, C) showed that purifying selection acts on the four Q-encoding codons. In particular, the downward columns in Fig. 1B indicate that the four Qs, similarly to the first 17 aa, are under purifying selection. Despite the possible limitations of aligning sequences bearing different CAG lengths, the three different approaches (FUBAR, SLAC, and FEL) suggest consistent purifying selection acting also on the polyQ tract of the mammalian sequences with $Q \geq 4 \quad(n=74$ species) (Fig. 1C, S2B, D and Supplementary dataset S5). Furthermore, possible evolutionary scenarios (negative, neutral, and positive selection) were evaluated by FUBAR method on the HTT exon 1 as a whole (Fig. S3). The distribution of substitution rates probability in Fig. S3A, B displayed that the higher (red) probability for $H T T$ exon1 fell exclusively into the negative selection area (Fig. S3A, B) indicating that $H T$ exon 1 , as a whole, is under purifying selection.

This is the first time a wide set of orthologs has been taken into consideration to represent aspects of $\mathrm{HTT}^{\prime} \mathrm{s}$ unique evolutionary history, among the polyQ-containing proteins under selective pressures [45], and to demonstrate that $H T T$ exon 1 as a whole and its polyQ tract underwent purifying selection.

\section{The HTT polyQ tract has been subjected to the strongest selective pressures throughout evolution compared to other polyQ-containing genes}

In order to investigate whether similar selective pressures might also act on the other polyQ, we tested the polyQ stretch of Ataxin3 (ATXN3) which is associated with the second most common polyQ disease, Spinocerebellar ataxia type 3 (SCA3) [46]. To this end, we generated an alignment of the ATXN3 polyQ locus from 53 taxa for which both ATXN3 and HTT sequences were available, thus sharing phylogenetic relationships and timescales (Supplementary datasets S6-8). Figure S4A, B show, for the same taxa, the distribution of substitutions around the polyQ region of ATXN3 and $\mathrm{HTT}$, respectively. While 53 synonymous and 43 nonsynonymous substitutions were found in the ATXN3 polyQ stretch, also in this sample only 21 synonymous substitutions were observed in the HTT polyQ stretch. FUBAR identified that $12.5 \%$, for ATXN3, and $44.4 \%$, for $\mathrm{HTT}$, of Qs were under significant purifying selection (Fig S4C, D). This suggests that the HTT polyQ tract is under stricter selective pressures compared to the ATXN3 polyQ locus.

We then performed selective pressure analysis on the polyQ tract of the other seven brain disease-related proteins [47], and of two proteins not-associated with any disease (POU6F2 and ZNF384) (Fig. 1D and S5 and Supplementary datasets S9-17). Comparative SLAC analysis revealed that the HTT polyQ tract is the first in terms of low presence of synonymous and nonsynonymous substitutions (Fig. 1D, and S4A, B and S5A). The analysis also revealed that this tract has one of the highest percentages of $\mathrm{Q}$ residues under significant purifying selection, ranking third after POU6F2 and TBP (Fig. 1D and S4C, D and S5B).

Recent findings showed that HD pathogenesis is influenced by the size of the uninterrupted CAG repeat sequence [6]. In particular, it is known that the stretch of CAG in the human $H T T$ gene is interrupted by a penultimate CAA codon. Studies from the last few years have demonstrated that the loss of this interruption, or the duplication of the ultimate CAA-CAG codons, are associated with an earlier or later-respectively-age of disease onset [6, 7], suggesting CAG purity is a causal driver of CAG somatic instability in $\mathrm{HD}[6,7]$. We therefore decided to explore mammalian genomes to compare HTT CAG purity and length with those of other genes encoding polyQ-containing proteins. We retrieved from the Ensembl database 248 sets of mammalian protein orthologs, containing at least one tract of four or more consecutive Qs for which length variability among mammalian species is observed, and collectively obtained 367 polyQ tracts because some proteins bear multiple polyQ tracts (Fig. S6A). For each individual polyQ tract, we calculated the $z$-scores (see methods) for (i) length of polyQ (LQ), (ii) longest uninterrupted CAG repeat (LNI), and (iii) polyQ tract "purity" (proportion of CAG/ CAA codons) with respect to orthologous sequences. We found that the human $\mathrm{HTT}$ polyQ tract behaved as an outlier both in terms of LQ (Fig. S6B and Supplementary dataset S18) and LNI (Fig. S6C and Supplementary dataset S18), as shown by its position in the right-most end tail of the distributions. The human HTT polyQ tract also displays an exceptionally low presence of CAA "impurity" compared to polyQ stretches of other proteins (Fig. S6D and Supplementary dataset S18). Indeed, human HTT displays the highest length and purity of the CAG tract compared to orthologs of known polyCAG/CAA disease-associated genes (Fig. 1E), such as ATXN3 (as also expected from the previous polyQ conservation analysis; Fig. S4A, C).

These results indicate that strong purifying selection acts on the CAG/CAA repeat region of the $H T T$ gene to maintain its capacity to encode glutamine residues. Our data also indicates that the human HTT polyQ encoding tract stands out for its length and purity of CAG codons with respect to other polyQ tracts in mammals. In conclusion, this level of conservation and purity in the HTT polyQ could have paved the way for its evolutionary expansion [7, 48-50].

\section{The structure of a de novo identified HTT pseudogene confirms that purifying selection acts on the CAG repeat region}

In our sequencing effort, we also serendipitously identified a processed HTT pseudogene ( $p H T T)$ in the marmoset Callithrix jacchus, which had escaped previous identification [51], and whose sequence allows the description of the mutational dynamics of this DNA sequence in the absence of selective constraints. Callithrix jacchus pHTT consists of the first nine HTT exons and lays within the third intron of the CNTNAP3 gene (Fig. 2A, B and Supplementary dataset S19). Three point-mutations (one non-synonymous) and several indels, including a frameshift 


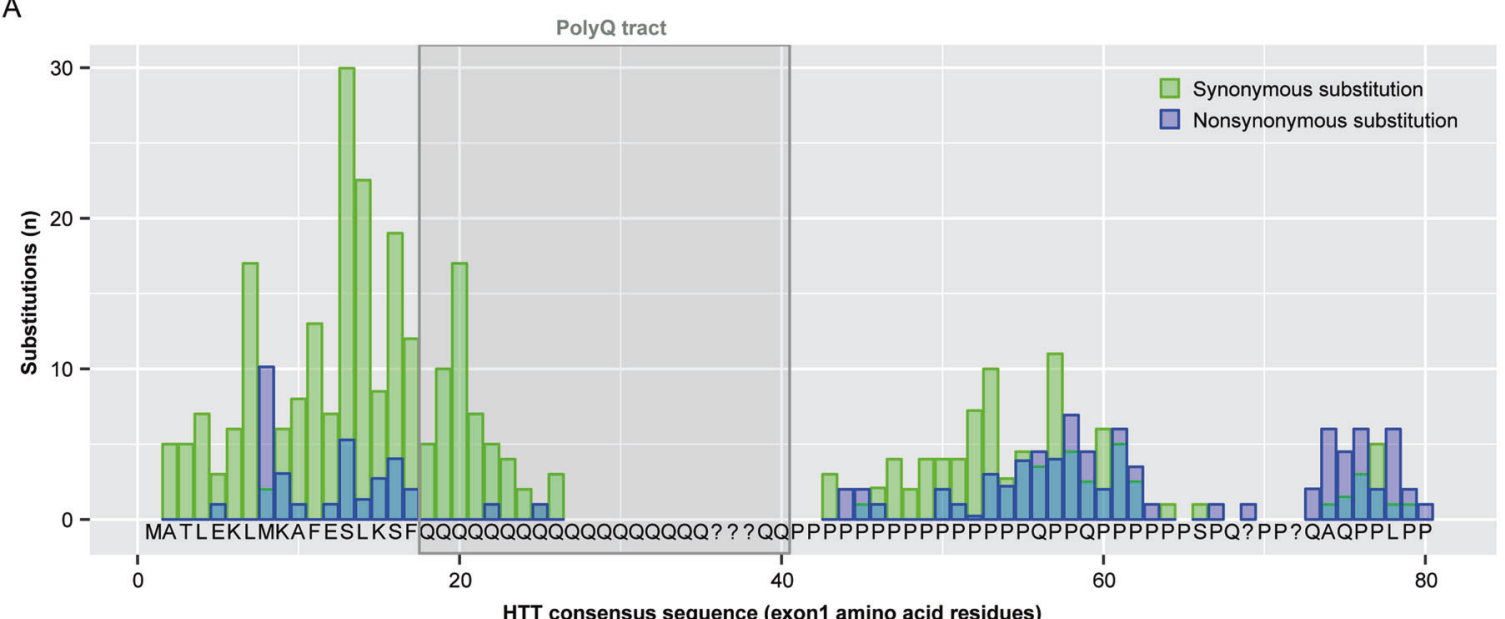

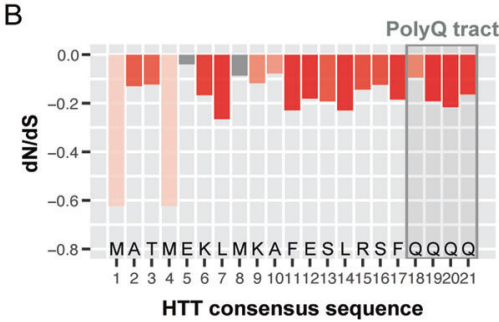

D
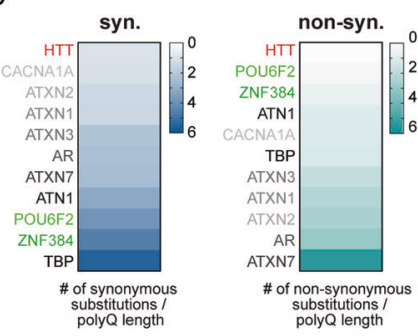

C

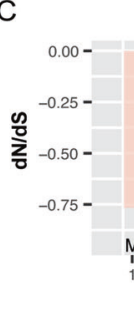

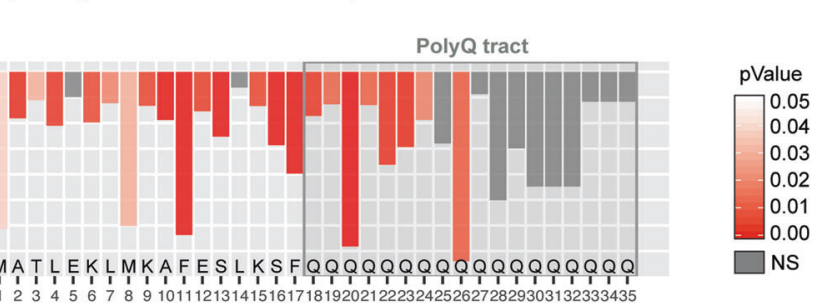

E

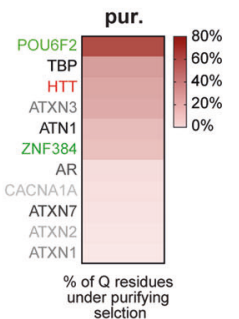

\begin{tabular}{lcccc}
\hline \multicolumn{1}{c}{ Gene } & Ensembl Gene ID & Test 1 (LQ) & Test 2 (LNI) & Test 3 (PQ) \\
\hline HTT & ENSG00000197386 & 2.82 & 2.71 & 1.71 \\
ATN1 & ENSG00000111676 & 2.00 & 2.07 & 0.76 \\
AR & ENSG00000169083 & 0.33 & 0.45 & 0.33 \\
ATXN1 & ENSG00000124788 & 0.88 & 1.02 & 0.33 \\
ATXN2 & ENSG00000204842 & 1.31 & 0.85 & 0.58 \\
ATXN3 & ENSG00000066427 & 0.04 & -0.18 & -1.30 \\
CACNA1A & ENSG00000141837 & 1.91 & 1.91 & ND \\
ATXN7 & ENSG00000163635 & 2.10 & 2.22 & 0.38 \\
TBP & ENSG00000112592 & 1.93 & 1.78 & 0.92 \\
\hline
\end{tabular}

Fig. 1 Effects of natural selection on HTT exon1. A Synonymous and non-synonymous substitutions counted with the codon-based maximum likelihood method SLAC on the multiple alignment of 163 unique, non-redundant sequences from vertebrates $(n=158)$, and basal species $(n=5)$. A time-tree was used as backbone for calculations. Synonymous (green) and non-synonymous (blue) substitution counts are shown for each codon (consensus sequence in the plot); the gray shaded box highlights the polyQ tract. B dN/dS ratios determined by FUBAR method for the multiple sequence alignment (MSA) subset of bony fishes, turtles, crocodiles, and birds ( $n=84$ species) where four glutamineencoding codons can be unambiguously aligned. Consensus sequence of $H T T$ exon 1 is shown for reference; the gray shaded box highlights the polyQ (4Q) tract. C dN/dS ratios determined by FUBAR method for the MSA of mammals $(Q \geq 4, n=74$ species), where the number of $Q$ encoding codons is variable. HTT N-terminal consensus sequence is shown for reference. The gray shaded box highlights the polyQ ( $Q \geq 4)$ tract. The orientation of peaks in plots $\mathbf{B}$ and $\mathbf{C}$ indicates the direction of selection (downward = purifying/negative; upward = diversifying/ positive); peaks height indicates the strength of selection (dN/dS values) and peak's color (shades of red) shows the statistical significance level. D Heatmaps showing comparison of the polyQ stretch conservations for nine polyQ disease-associated genes (HTT in red, others in gray scale) and two genes not associated with any type of diseases (POU6F2 and ZNF384, green) across taxa. The heatmaps display the value of synonymous substitutions over $Q$ length ratio (syn.), the value of non-synonymous substitutions over $Q$ length ratio (non-syn.) and the fraction of $Q$ residues under significative purifying selection (pur.). E Table showing the comparative analysis of the disease-associated polyQ proteins. Z-score values for the longest Q stretch (LQ), for the longest non-interrupted CAG interval (LNI) and for the CAG/CAA proportion (PQ) of the nine human disease-associated genes, extracted from the results of the three analyses (test 1, 2, and 3) described in Fig. S6B-D.

inducing insertion, were observed between the CAG tract of Callithrix jacchus $p H T T$ and $H T T$ (Fig. 2C). Numerous indels were also observed between the proline-rich domain (PRD) of the Callithrix jacchus $p H T T$ and other primate $H T T$ sequences (Fig. 2D). These observations likely reflect the absence of purifying selection acting on the pseudogene sequence and hint at the underlying mutability of sequences encoding the PRD. Interestingly, $\mathrm{pHTT}$ exon 1 in Callithrix jacchus is far more prone to point substitutions than the rest of the pseudogene exons, consistent with studies indicating that point substitution rates are higher around indel sites [52] (Fig. 2E and Supplementary dataset S20) and further attesting to the purifying selection acting on HTT exon1.

\section{The length and purity of the polyQ tract affects HTT neuronal} function

Since long polyQ tracts are enriched in genes with neuronal functions [53], and since HTT is implicated in neurogenesis $[18,54]$, we looked for evidence of polyQ activity itself in in vitro neural cell assays. We therefore genome-edited E14 mES cell line to knock-in $0,2,4,7,10,13$, or 17 Qs in the endogenous $H T T$ gene (Fig. 3A, B). 7Qs are found in the mouse HTT protein $([55,56]$ and Fig. S1). First, we integrated a Recombinase-Mediated Cassette Exchange (RMCE) cassette at the exon 1 locus by CRISPR-driven homologous recombination, in parallel with the deletion of the other $H T T$ allele to create RMCE/- cells. Flp-mediated exchange of 
A

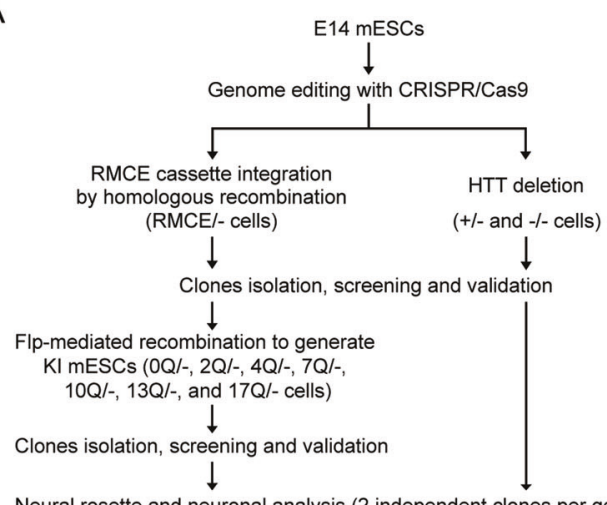

Neural rosette and neuronal analysis (2 independent clones per genotype)

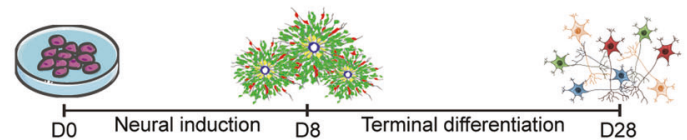

C
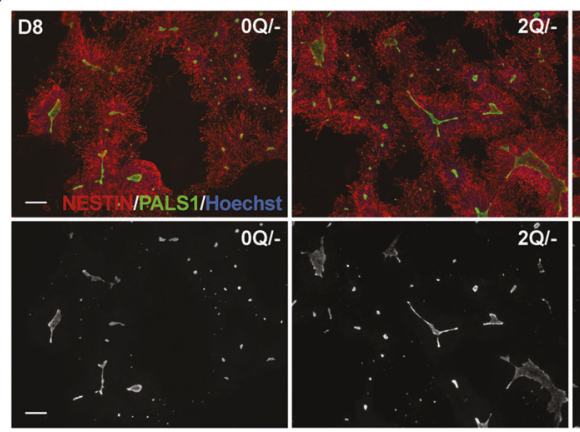

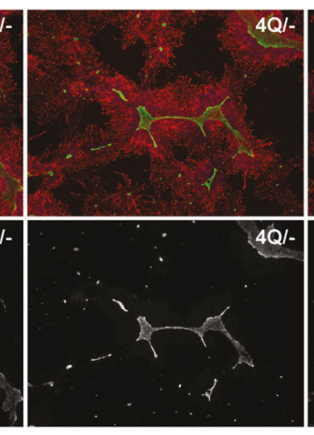

B

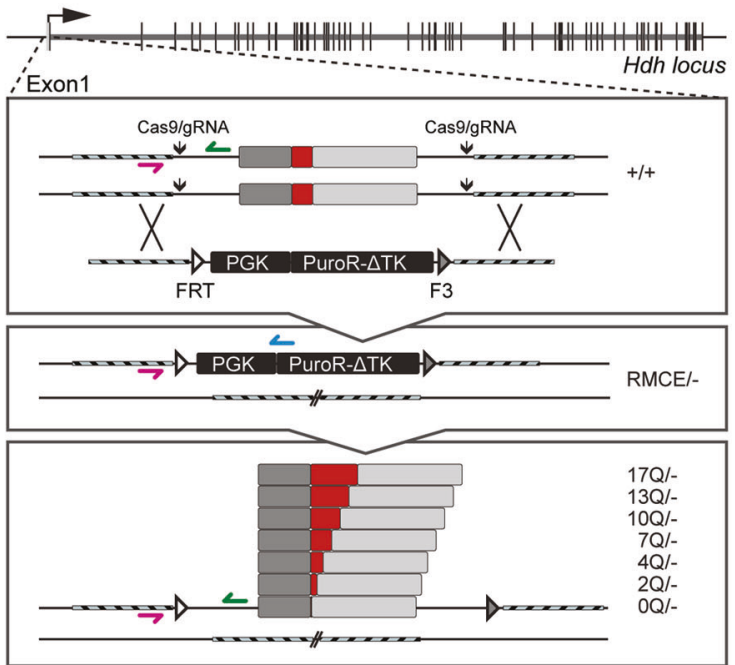

D
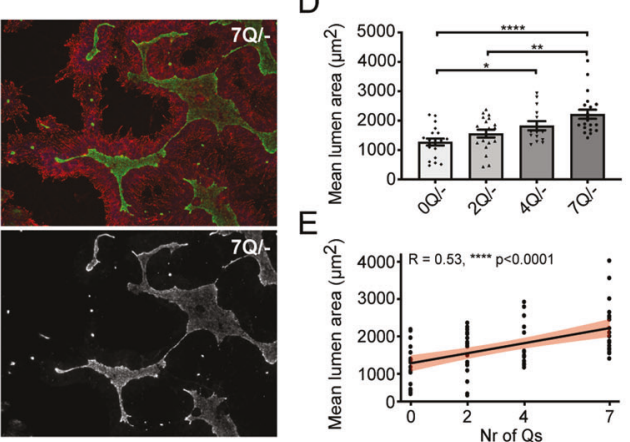

Fig. 3 PolyQ tract impacts the formation of neural structures. A Pipeline for the generation and characterization of HTT knock-in and knockout E14 mESCs. B Genome editing strategy used to produce knock-in mESCs. CRISPR/Cas9 with two gRNAs, was used to insert the RMCE cassette replacing the exon 1 on one allele and to delete HTT exon 1 on the other allele, thus generating the RMCE/- cell line. The RMCE cassette contains the positive/negative selectable marker PuroR- $\Delta$ TK, under PGK promoter, flanked by FRT and F3 recombination sites, which were used to direct the integration of modified exons 1 with $0,2,4,7,10,13$, and $17 \mathrm{Q}$ repeats by Flp recombinase. gRNAs and oligos sequences are reported in Supplementary dataset S23. C Representative images of rosette/lumen phenotype in 0Q/-, 2Q/-, 4Q/-, and 7Q/cells stained for PALS1 and NESTIN at day 8 of neural induction. D Mean lumen area $\left(\mu \mathrm{m}^{2}\right)$ of $0 \mathrm{Q} /-, 2 \mathrm{Q} /-, 4 \mathrm{Q} /-$, and $7 \mathrm{Q} /-\mathrm{cell}$ cultures. ${ }^{*} P<$ $0.05,{ }^{* *} P<0.01,{ }^{* * * *} P<0.0001$, one-way ANOVA test followed by Tukey. E Linear regression analysis and confidence interval between $\mathrm{Q}$ length and lumen size in $0 \mathrm{Q} /-, 2 \mathrm{Q} /-, 4 \mathrm{Q} /-$, and $7 \mathrm{Q} /-$ cell cultures. Pearson correlation coefficients $(\mathrm{R})$ and $P$-values are reported on the plots. Data in $\mathbf{D}$ and $\mathbf{E}$ are expressed as mean \pm SEM from $n \geq 4$ independent experiments. Each dot represents the mean lumen area per well testing a pool of two clones for each edited cell line (see Supplementary dataset S25 for raw data). The scale bars correspond to $50 \mu \mathrm{m}$.

first 548aa portion carrying 0, 2, 4, or 7 Qs (Fig. S10A, B, D). Differently from E14 mES cells, parental R1 cells generate single rosettes that are more easily definable, allowing the measurement of two additional parameters: rosette area and the percentage of NESTIN + cells within the rosettes. Even here, Q tract length was found to correlate with rosette formation potential, as well as with lumen and rosette areas (Fig. $\mathrm{S} 10 \mathrm{E}-\mathrm{H}$ ). In particular, the $0 \mathrm{Q}$ line generated fewer neural progenitors organized in rosettes and reduced rosette and lumen size, similar to unmodified R1 Hdh ${ }^{\text {ex4-5 }}$ cells, and contrary to the $7 Q$ cells where the wild-type rosetteforming condition was restored (Fig. S10E, I-K). Furthermore, interruption of the $\mathrm{Q}$ stretch with a non-synonymous CCG substitution, encoding proline in the 4th position of the polyQ tract (Q3PQ3 cells; Fig. S10A, C, D), reduces the amount of organized progenitors and generates smaller rosettes and lumens compared to $\mathrm{R} 1 \mathrm{Hdh}^{+/+}$and $7 \mathrm{Q}$ cells (Fig. S10M-P). In contrast, a CAA synonymous substitution in the same codon (Fig. S10A, C, D) does not affect the rosette phenotype (Fig. S10M-P). We conclude that, also in conditions of overexpression, a $7 \mathrm{Q}$ tract is more effective in promoting rosette formation than $0 \mathrm{Q}$, and that interrupting the purity of $\mathrm{Q}$ residues with only one proline disturbs rosette formation. In contrast, the known anti-apoptotic activity of $\mathrm{HTT}$ [58] was not affected in $0 \mathrm{Q}$ cells, which behaved as $7 \mathrm{Q}$ and $\mathrm{Hdh}^{+/+}$cells (Fig. S10L), indicating that the anti-apoptotic activity of $\mathrm{HTT}$ does not reside within its polyQ tract.

To test whether small variations in the non-pathogenic polyQ size can have an impact on the phenotype at more advanced stages of neuronal differentiation, we subjected E14 mES cellderived $0 \mathrm{Q}, 2 \mathrm{Q}, 4 \mathrm{Q}$, and $7 \mathrm{Q}$ neural progenitors to pan-neuronal terminal differentiation until day 28 (Fig. 3A). Overall, terminally differentiated neuronal populations are more reminiscent of cortical cells regardless of $Q$ length (Fig. S11A). We found that the level of the late-stage neuronal marker MAP2 in $7 Q$ neurons is approximately 1.5 -fold higher than in $0 \mathrm{Q}$ neurons (Fig. S11B, C). Moreover, an increase in the percentage of P27 and NEUN immunoreactive (post-mitotic) cells was found in $7 Q$ compared to $0 \mathrm{Q}$ neurons (Fig. S11B, E, G). Fig. S11 also shows a positive correlation between the expression of late-stage neuronal markers (MAP2, P27, and NEUN) and the number of Qs, especially from 0 to $7 \mathrm{Q}$, therefore providing further measurement of the ability of longer polyQ tract to affect biological parameters associated with neuronal differentiation (Fig. S11D, F, H). 
A

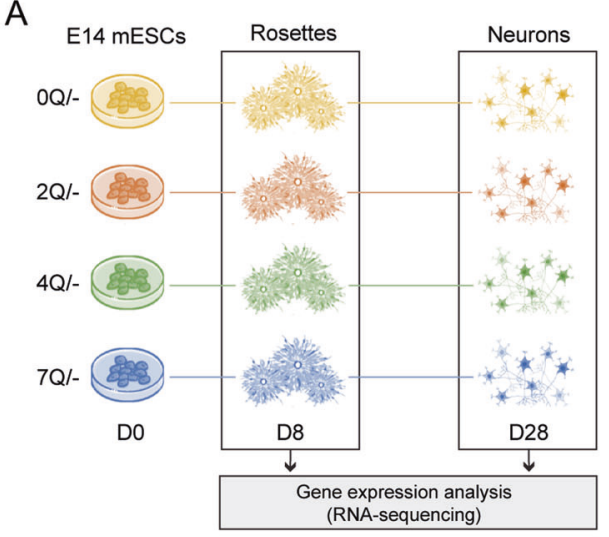

B

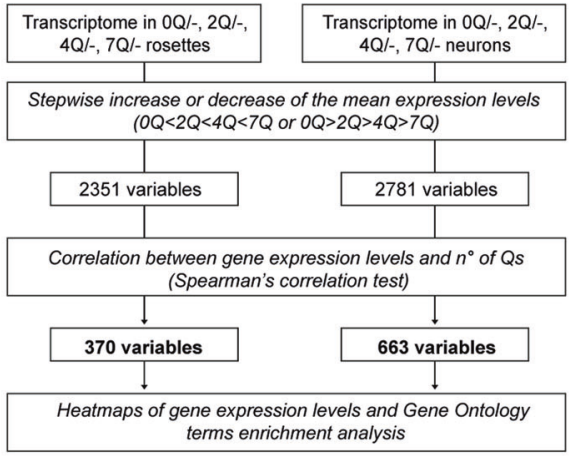

C

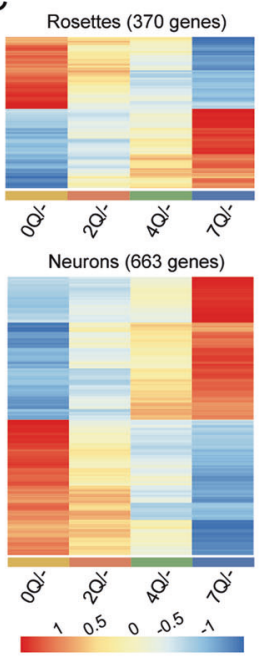

E

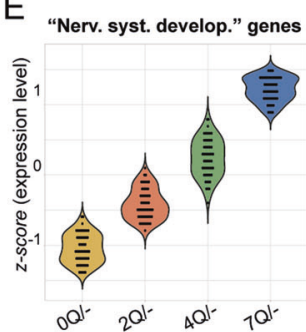

D

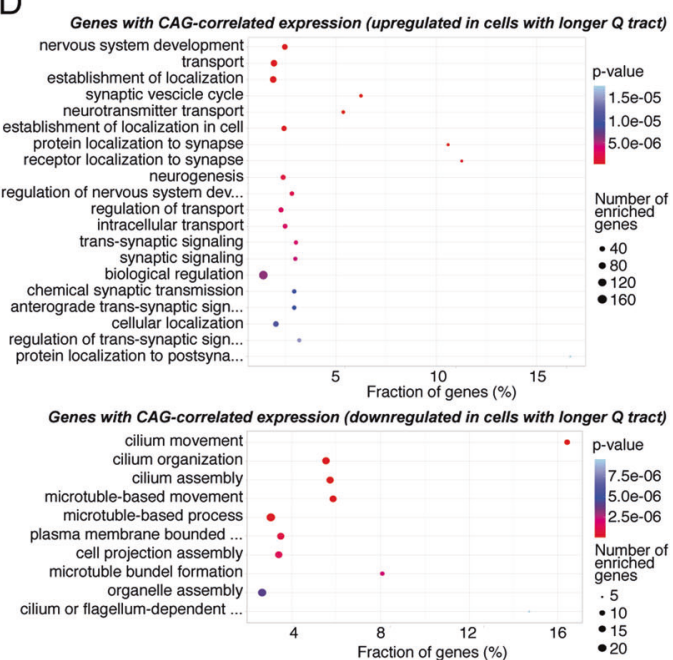

$F$

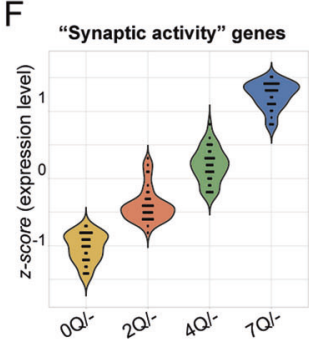

Fig. 4 PolyQ length-sensitive influence in transcriptional programs governing in vitro neuronal differentiation. A Experimental scheme of gene expression analysis by RNA-seq at rosette and neuron stage among 0, 2, 4, and $7 Q$ cell lines. B Schematic pipeline of whole transcriptomics-based analysis designed to identify genes whose expression increase or decrease stepwise $(0 Q>2 Q>4 Q>7 Q$ or $0 Q<2 Q<$ $4 Q<7 Q$ ) and linearly (Spearman's correlation) with CAG-length in 0,2, 4, and $7 Q$ transcriptomes. C Heatmaps of the expression levels for genes identified applying the pipeline depicted in B. The data was normalized and scaled. D GO dot plots of the top significant GO terms (ranked on their p-value from a classic Fisher test) of the genes, identified in B, which are upregulated (upper panel) or downregulated (bottom panel) in neurons with increasing number of Qs. For the full lists of DEGs see Supplementary dataset S21. E-G Violin plots of genes from B showing significant $Q$ length-correlated expression selected for nervous system development (GO:0007399 term) (E), synaptic activity (GO:0099537, GO:0050808, GO:0035418 terms) (F) or cilium formation processes (GO:0060271 and GO:0044782 terms) (G) in 0Q, 2Q, 4Q, and $7 \mathrm{Q}$ neurons. Each dot represents the $z$-score value for each gene ( $z$-score values were calculated by subtracting the population mean of expression levels from the sample mean of the raw expression levels and, then, dividing by the population standard deviation).

All together, these experiments reveal that the length and purity of the HTT polyQ tract impacts aspects of neuronal cell biology in vitro.

\section{The HTT polyQ tract regulates pivotal genes linked to early-to- late neural development}

To explore the molecular changes that occur as a consequence of small length variations in the HTT polyQ tract which are reflected by the observed neural phenotypes (Fig. 3 and S11), we performed transcriptomic analyses of two cardinal stages of neuronal differentiation of $0,2,4$, and $7 \mathrm{Q}$ knock-in E14 mES cell lines (Fig. 4A). Initially, we conducted a comprehensive investigation of the CAG length-driven molecular changes, considering the whole transcriptome of all lines at early (rosettes) and later (neurons) stage of cell differentiation (Fig. 4B). We found that 2351, for rosettes, and 2781 genes, for neurons, showed a stepwise increase $(0 \mathrm{Q}>2 \mathrm{Q}>4 \mathrm{Q}>7 \mathrm{Q})$ or decrease $(0 \mathrm{Q}<2 \mathrm{Q}<4 \mathrm{Q}<7 \mathrm{Q})$ in expression levels (Fig. 4B). Correlation analysis revealed that 370 genes, for rosettes, and 663 genes, for neurons, exhibit a significant correlation between the number of $Q s$ and their expression levels (Fig. 4B, C). Notably, functional enrichment analyses (Fig. 4D) of genes showing a $\mathrm{Q}$ length-correlated expression at the later stage of differentiation revealed enriched gene ontology (GO) terms related to neuronal aspects, such as nervous system development (Fig. 4E), synaptic activity (Fig. 4F) and cilium formation (Fig. 4G), supporting the hypothesis that the non-pathogenic $Q$ length in $\mathrm{HTT}$ affects parameters related to neuronal differentiation.

We then identified the main actors involved in $\mathrm{Q}$ length-related phenotypes by performing pairwise comparisons among all the cell lines at two time-points, rosettes and neurons (Fig. 5A and S12), with a specific focus between $0 Q$ and $7 Q$ lines. We detected differentially expressed genes (DEGs) between $0 \mathrm{Q}$ and $7 \mathrm{Q}$ genotypes at both rosette and neuron stages, with a higher number of DEGs in the latter (Fig. $5 \mathrm{~B}, \mathrm{C}$ ). A total of $41 \mathrm{DEG}$ in $0 \mathrm{Q}$ versus $7 \mathrm{Q}$ rosettes are enriched in GO terms related to neuro-development (Fig. 5B, D, E and Supplementary dataset S21). Among these genes, we found NNAT, PLAGL1 (also known as ZAC1), LBX1 and OLIG2, which are implicated in the organization of neuroepithelial progenitors during neurogenesis (Fig. 5B) [59-64]. We confirmed by qPCR that $0 Q$ neural progenitors show abnormally elevated expression of neurogenic factors NNAT and PLAGL1 (Fig. 5H), whose dysregulation may affect the ability of $\mathrm{OQ}$ cells to correctly generate neural rosettes.

When $0 \mathrm{Q}$ and $7 \mathrm{Q}$ cultures were compared at the more mature neuronal stage, 145 DEGs were detected. Among them, we found in the $\mathrm{OQ}$ line a deregulation of genes implicated in neuronal maturation, cell fate, and synaptic activity (Fig. 5C, F, G and Supplementary dataset S21), reflecting the increased capacity of the $7 \mathrm{Q}$ line of acquiring more mature neuronal signatures. Among neuronal maturation DEGs, we confirmed by qPCR that NNAT and PLAGL1 were elevated in $0 \mathrm{Q}$ compared to $7 \mathrm{Q}$ neurons (Fig. $5 \mathrm{H}$ ) with 
A

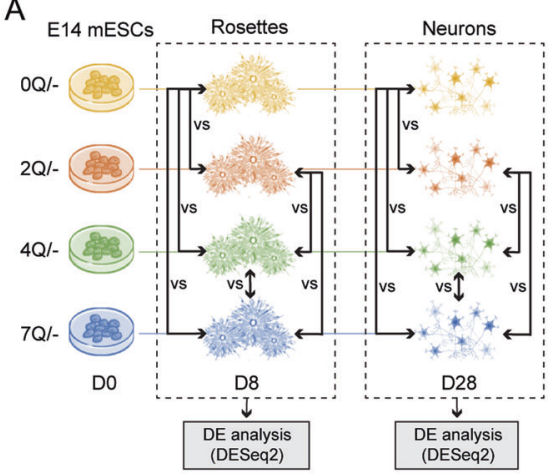

B

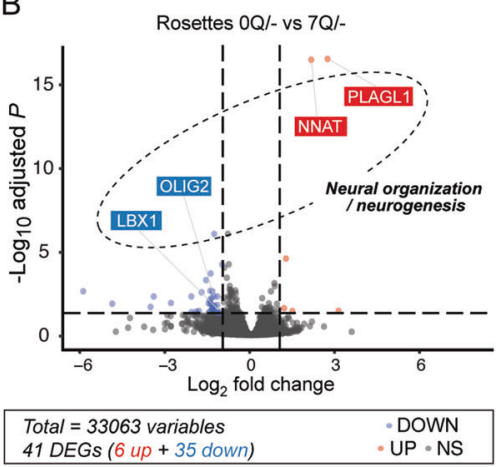

C

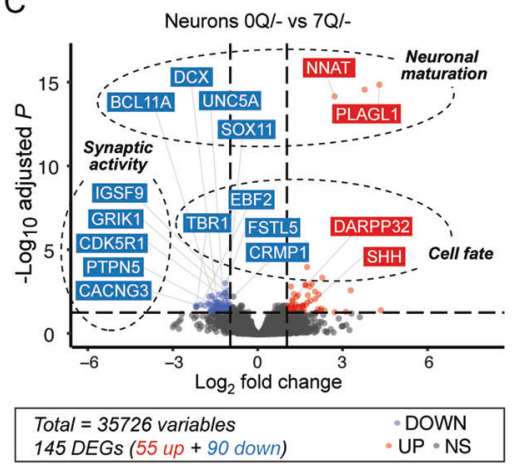

D

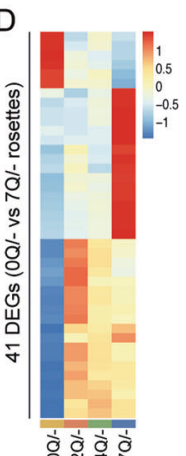

E

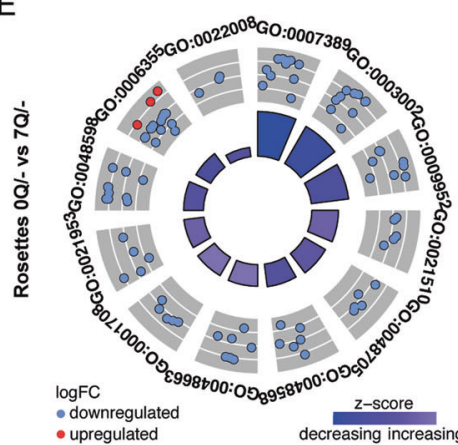

\begin{tabular}{|c|c|c|}
\multicolumn{1}{|c|}{ ID } & Description & $\begin{array}{c}\text { Gene } \\
\text { fraction }\end{array}$ \\
\hline GO:0007389 & pattern specification process & $11 / 467$ \\
\hline GO:0003002 & regionalization & $10 / 372$ \\
\hline GO:0009952 & anterior/posterior pattern specificif.. & $8 / 230$ \\
\hline GO:0021510 & spinal cord development & $5 / 102$ \\
\hline GO:0048705 & skeletal system morphogenesis & $6 / 262$ \\
\hline GO:0048568 & embryonic organ development & $7 / 490$ \\
\hline GO:0048663 & neuron fate commitment & $4 / 81$ \\
\hline GO:0001708 & cell fate specification & $4 / 89$ \\
\hline GO:0021953 central nervous system neuron dif.. & $5 / 223$ \\
\hline GO:0048598 & embryonic morphogenesis & $7 / 634$ \\
\hline GO:0006355 regulation of transcription, DNA-te.. & $12 / 2776$ \\
\hline GO:0022008 & neurogenesis & $7 / 1768$ \\
\hline \multicolumn{3}{|c|}{} \\
\hline
\end{tabular}

$\mathrm{H}$

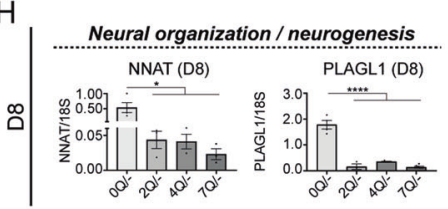

Neuronal maturation
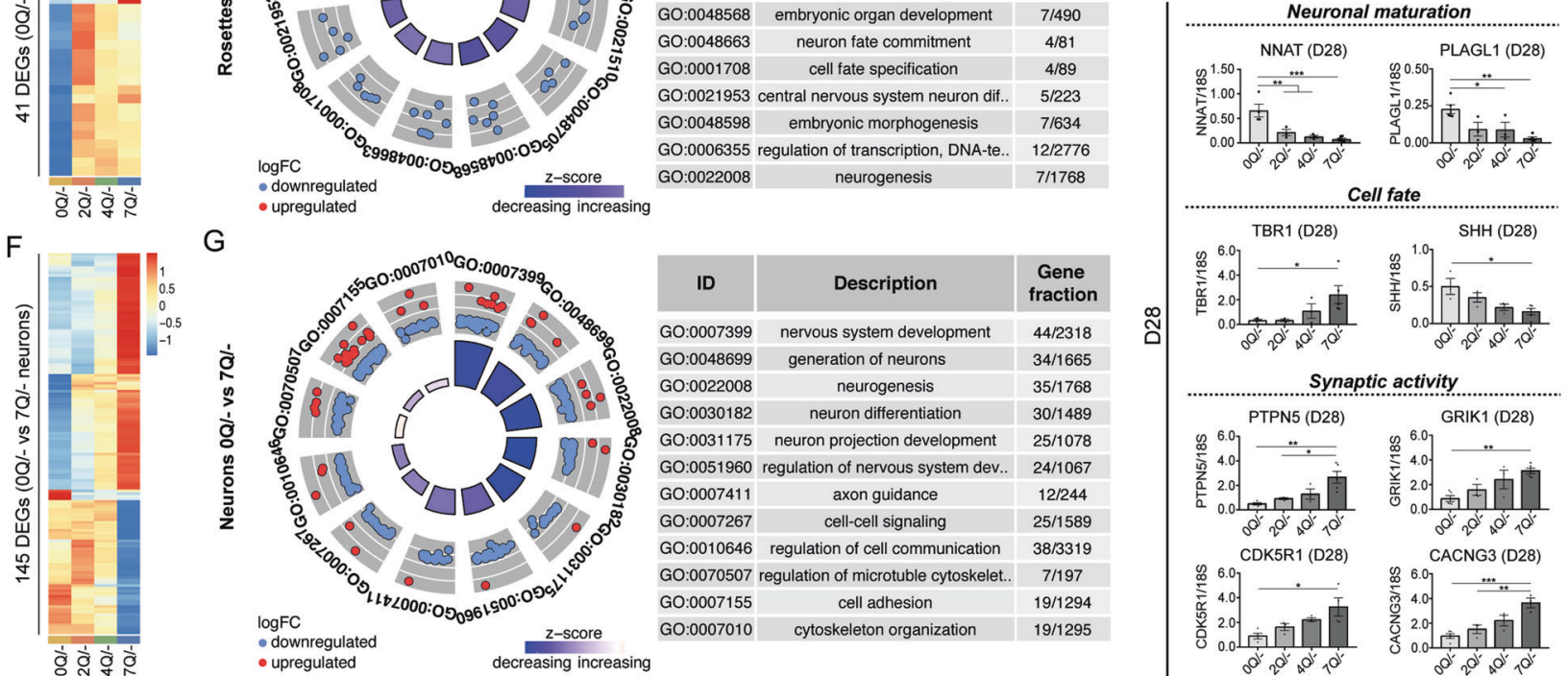

G

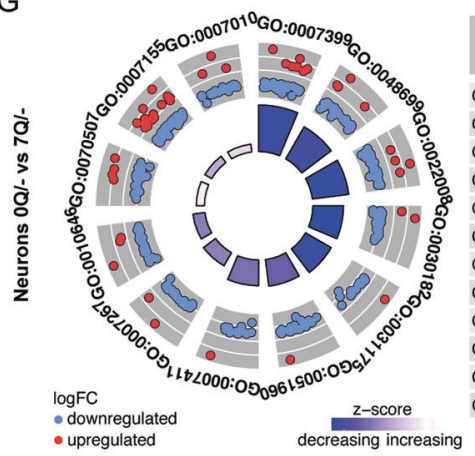

\begin{tabular}{|c|c|c|}
\hline \multicolumn{1}{|c|}{ ID } & Description & $\begin{array}{c}\text { Gene } \\
\text { fraction }\end{array}$ \\
\hline GO:0007399 & nervous system development & $44 / 2318$ \\
\hline GO:0048699 & generation of neurons & $34 / 1665$ \\
\hline GO:0022008 & neurogenesis & $35 / 1768$ \\
\hline GO:0030182 & neuron differentiation & $30 / 1489$ \\
\hline GO:0031175 & neuron projection development & $25 / 1078$ \\
\hline GO:0051960 & regulation of nervous system dev. & $24 / 1067$ \\
\hline GO:0007411 & axon guidance & $12 / 244$ \\
\hline GO:0007267 & cell-cell signaling & $25 / 1589$ \\
\hline GO:0010646 & regulation of cell communication & $38 / 3319$ \\
\hline GO:0070507 regulation of microtuble cytoskelet.. & $7 / 197$ \\
\hline GO:0007155 & cell adhesion & $19 / 1294$ \\
\hline GO:0007010 & cytoskeleton organization & $19 / 1295$ \\
\hline
\end{tabular}

Fig. 5 RNA-seq analyses reveal the HTT polyQ-related molecular pathways underlying different signatures in the early-to-late generation of neurons among 0, 2, 4 and $7 Q$ cell lines. A Experimental scheme of gene expression analysis by RNAseq in which pairwise comparisons at rosette and neuron stage among 0, 2, 4, and 7Q (E14) cell lines were performed. B, C Volcano plots of DEGs between 0Q vs. $7 \mathrm{Q}$ at rosettes (B) and neuron (C) stage. Significant DEGs (adjusted $P$-value $<0.05$ ) are labeled in red (for up-regulation with logFC $>1$ ) or blue (for downregulation with logFC $<-1$ ). The classes of genes subsequently validated by qPCR analysis are reported on the plot. $\mathbf{D}$ Heatmap of gene expression levels for significant DEGs between $0 \mathrm{Q}$ vs. $7 \mathrm{Q}$ rosettes (41 genes). The data was normalized and scaled. E Gene-ontology (GO) circle plot displaying the top significant (ranked on their $P$-value) non-redundant GO terms of the DEGs between rosettes $0 \mathrm{Q}$ vs. $7 \mathrm{Q}$. Within each selected GO term the outer chart shows the distribution of the logFC of the individual assigned genes from higher (outer layer) to lower (inner layer). Genes are represented as red (upregulated) and blue (downregulated) circles. The height of the inner bars represents the $P$-value of the $\mathrm{GO}$ term whereas its color indicates the $z$-score (see "Methods" section). F Heatmap of gene expression levels for significant DEGs between $0 \mathrm{Q}$ vs. $7 Q$ neurons (145 genes). The data was normalized and scaled. G GO circle plot displaying the top significant (ranked on their $P$-value) nonredundant GO terms of the DEGs between neurons $0 Q$ vs. 7Q. For the details of the plot see $\mathbf{E}$. $\mathbf{H}$ qPCR validation analyses of significant DEGs between $0 Q$ vs. $7 Q$ cell lines at rosette (D8) and neuron (D28) stage. Bar plots display the mean values \pm SEM from $n \geq 3$ biological replicates; ${ }^{*} P<0.05$, ${ }^{* *} P<0.01,{ }^{* * *} P<0.001$, ${ }^{* * * *} P<0.0001$, one-way ANOVA test followed by Tukey. Values of the logFCs are scaled. For the full lists of DEGs see Supplementary dataset S21.

possible consequences for terminally differentiated $\mathrm{OQ}$ neurons $[62,63]$. We also found increased expression of the typical dorsal marker TBR1 (Fig. 5H) (as well as of other cortical markers such as EBF2, FSTL5, and CRPM1; Fig. 5C) and reduced expression of the ventral marker SHH (Fig. $5 \mathrm{H}$ ) in $7 \mathrm{Q}$ neurons. Finally, we validated a third class of DEGs implicated in synapse activity and dendrite outgrowth (such as CDK5R1, PTPN5, GRIK1, and CACNG3) whose expression was found to be increased in $7 \mathrm{Q}$ compared to $0 \mathrm{Q}$ neurons (Fig. 5H).
Collectively, these results show that the HTT polyQ tract is biologically active and seems to orchestrate key factors of in vitro neural organization, cell fate, and neuronal maturation processes.

\section{DISCUSSION}

In this study, by exploring vertebrate genomes, we provide the strongest evidence to date that the CAG repeats in the HTT gene are the product of purifying selection against deleterious 
mutations. Purifying natural selection is a hallmark of functional biological sequences and the non-pathogenic CAG-polyglutamine repeats in HTT must confer advantageous traits to the protein. Consistent with this, we discovered that the HTT non-pathogenic polyQ tract has neuronal activities. In line with our finding, previous studies have shown that $Q$ deletion in mice causes cognitive deficits in specific tests [65]. Moreover, studies conducted in humans, suggest that an increase in the number of CAG repeats in HTT-below the disease threshold-confers positive changes in human brain structure and general intelligence [22-24] as well as improvements in cognitive performance [25].

In our study we found a positive correlation between the length of the non-pathogenic polyQ tract and phenotypes, demonstrating that very small variations in the number of CAG repeats are sufficient to mediate the transcriptional response of differentiating neuronal cells. Our transcriptome analyses propose that polyQ tract length influences neural phenotypes by modulating the expression of genes involved in neural organization, neuronal identity, synapse maturation, and cilium formation. Of note, we found that an elongating CAG tract positively affects genes involved in vesicle trafficking as well as in synapse architecture and activity, while it negatively affects genes involved in ciliogenesis. These findings consistently emphasize some of the known physiological functions of $\mathrm{HTT}[8,9]$ and corroborate the hypothesis that longer physiological polyQ tract could possibly generate more mature, more connected and more functional neurons. Curiously, many of the processes - that we found to be regulated by the polyQ tract in our in vitro models-are the same ones on which natural selection usually operates to promote the evolution of neural circuits [66]. Therefore, we hypothesize that evolution has selected for longer CAG HTT stretches since they carry advanced neuronal activities.

Overall, our findings demonstrate that the HTT polyQ tract is functionally active in our neural cell assays and that small size fluctuations in the number of Qs influence its biological activity. The CAG repeat in the HTT gene may therefore have been selected throughout evolution to act as a genetic modulator of brain development and function [15].

Our study provides experimental evidence of the neural activity of the HTT physiological polyQ tract, supporting the hypothesis that simple sequence repeats, as CAG repeats, play a fundamental role in evolution by producing the variability needed to enhance changes in neuronal/brain development $[15,67,68]$. Previous works suggest that CAG-encoded polyQ tracts are enriched in genes with neuronal functions [53] while polyQ diseases always exhibit elements of neurological dysfunction [69], consistent with the hypothesis that these three-nucleotide repeats might indeed be physiologically relevant.

$\mathrm{HTT}$ is highly expressed in brain and testis and it is also found in other non-neuronal tissues [70, 71]. The CAG tract length may therefore also affect activities of HTT in other tissues. For example, in vivo studies have shown that the protein is implicated in spermatogenesis by regulating translation and DNA packaging in the testis [72]. Whether natural selection is operating more broadly, not exclusively on CAG-dependent neuronal phenotypes, remains to be elucidated.

The molecular machinery responsible for the elongation of the HTT CAG tract is unknown. Evidence that a pure CAG tract leads to instability and expansion of the tract, while CAA interruptions leads to a more stable tract [7] allows us to hypothesize that this mechanism may also occur during the expansion observed during evolution. There may also be trans-acting modifiers, such as genes linked to the control of DNA replication and repair [73] that have co-evolved with $\mathrm{HTT}$, allowing for the expansion of the CAG tract in mammals especially in humans, as seen in the disease [74]. Hence, the pathological CAG repeat found in patients may represent the side effect of an evolutionary path that aims at maintaining the purity of an elongating and beneficial CAG repeat in the human gene. In this complex scenario, human $\mathrm{HTT} Q$ repeat lengths may reflect a delicate balance between the evolutionary advantages of having a pure and longer tract and the detrimental effects of an extreme expansion.

Our findings may lead to a paradigm shift in our view of a rare genetically dominant adult-onset neurological disease whose underlying genetic trait has not been selected against. Historically, it has been assumed that the preservation of an elongated repetitive tract in the human state was due to a simple lengthdependent mutational bias towards longer alleles [11]. Our study, on the other hand, advocates that the HTT polyQ tract has been selected throughout evolution, possibly to pursue its functional neural purpose.

\section{DATA AVAILABILITY}

The authors declare that all data supporting the findings of this study are available within the paper in the main text or the supplementary materials. Bulk RNA-seq data have been deposited in the ArrayExpress database at EMBL-EBI (https://www.ebi.ac. uk/arrayexpress/) under accession no. E-MTAB-9378.

\section{REFERENCES}

1. Reiner A, Albin RL, Anderson KD, D'Amato CJ, Penney JB, Young AB. Differential loss of striatal projection neurons in Huntington disease. Proc Natl Acad Sci USA. 1988;85:5733-7.

2. O'Donovan, MC. A novel gene containing a trinucleotide repeat that is expanded and unstable on Huntington's disease chromosomes. The Huntington's Disease Collaborative Research Group. Cell. 1993;72:971-83.

3. Rosas HD, Salat DH, Lee SY, Zaleta AK, Pappu V, Fischl B, et al. Cerebral cortex and the clinical expression of Huntington's disease: complexity and heterogeneity. Brain 2008;131:1057-68.

4. Davies SW, Turmaine M, Cozens BA, DiFiglia M, Sharp AH, Ross CA, et al. Formation of neuronal intranuclear inclusions underlies the neurological dysfunction in mice transgenic for the HD mutation. Cell 1997;90:537-48.

5. Bañez-Coronel M, Porta S, Kagerbauer B, Mateu-Huertas E, Pantano L, Ferrer I et al. A pathogenic mechanism in Huntington's disease involves small CAGrepeated RNAs with neurotoxic activity. PLoS Genet. 2012;8:e1002481.

6. Genetic Modifiers of Huntington's Disease (GeM-HD) Consortium. CAG repeat not polyglutamine length determines timing of Huntington's disease onset. Cell. 2019;178:887-900.

7. Wright GEB, Collins JA, Kay C, McDonald C, Dolzhenko E, Xia Q, et al. Length of uninterrupted $C A G$, independent of polyglutamine size, results in increased somatic instability, hastening onset of Huntington disease. Am J Hum Genet. 2019;104:1116-26.

8. Zuccato C, Valenza M, Cattaneo E. Molecular mechanisms and potential therapeutical targets in Huntington's disease. Physiol Rev. 2010;90:905-81.

9. Saudou F, Humbert S. The biology of Huntingtin. Neuron 2016;89:910-26.

10. Barron JC, Hurley EP, Parsons MP. Huntingtin and the synapse. Front Cell Neurosci. 2021;15:689332

11. Rubinsztein DC, Amos W, Leggo J, Goodburn S, Ramesar RS, Old J, et al. Mutational bias provides a model for the evolution of Huntington's disease and predicts a general increase in disease prevalence. Nat Genet. 1994;7:525-30.

12. Semaka A, Kay C, Doty CN, Collins JA, Tam N, Hayden MR. High frequency of intermediate alleles on Huntington disease-associated haplotypes in British Columbia's general population. Am J Med Genet B. 2013;162B:864-71.

13. Kay C, Collins JA, Miedzybrodzka Z, Madore SJ, Gordon ES, Gerry N, et al. Huntington disease reduced penetrance alleles occur at high frequency in the general population. Neurology 2016;87:282-8.

14. Kashi $Y$, King D, Soller M. Simple sequence repeats as a source of quantitative genetic variation. Trends Genet. 1997;13:74-8.

15. Fondon JW, Hammock EAD, Hannan AJ, King DG. Simple sequence repeats: genetic modulators of brain function and behavior. Trends Neurosci. 2008;31:328-34.

16. Kashi $Y$, King DG. Simple sequence repeats as advantageous mutators in evolution. Trends Genet. 2006;22:253-9.

17. Tartari M, Gissi C, Lo Sardo V, Zuccato C, Picardi E, Pesole G, et al. Phylogenetic comparison of huntingtin homologues reveals the appearance of a primitive polyQ in sea urchin. Mol Biol Evol. 2008;25:330-8.

18. Lo Sardo V, Zuccato C, Gaudenzi G, Vitali B, Ramos C, Tartari M, et al. An evolutionary recent neuroepithelial cell adhesion function of huntingtin implicates ADAM10-Ncadherin. Nat Neurosci. 2012;15:713-21. 
19. Haasl RJ, Payseur BA. Microsatellites as targets of natural selection. Mol Biol Evol. 2013:30:285-98.

20. Doolittle WF, Sapienza C. Selfish genes, the phenotype paradigm and genome evolution. Nature 1980;284:601-3.

21. Orgel LE, Crick FH. Selfish DNA: the ultimate parasite. Nature 1980;284:604-7.

22. Lee JK, Ding Y, Conrad AL, Cattaneo E, Epping E, Mathews K, et al. Sex-specific effects of the Huntington gene on normal neurodevelopment. J Neurosci Res. 2017;95:398-408.

23. Mühlau M, Winkelmann J, Rujescu D, Giegling I, Koutsouleris N, Gaser C, et al. Variation within the Huntington's disease gene influences normal brain structure. PLoS ONE. 2012;7:e29809.

24. Lee JK, Conrad A, Epping E, Mathews K, Magnotta V, Dawson JD, et al. Effect of trinucleotide repeats in the Huntington's gene on intelligence. EBioMedicine 2018;31:47-53.

25. Schultz JL, Saft C, Nopoulos PC. Association of CAG repeat length in the Huntington gene with cognitive performance in young adults. Neurology 2021;96: e2407-13.

26. Candiani S, Pestarino M, Cattaneo E, Tartari M. Characterization, developmental expression and evolutionary features of the huntingtin gene in the amphioxus Branchiostoma floridae. BMC Dev Biol. 2007;7:127.

27. Wicht $\mathrm{H}$, Lacalli TC. The nervous system of amphioxus: structure, development, and evolutionary significance. Can J Zool. 2005;83:122-50.

28. Hinman VF, Burke RD. Embryonic neurogenesis in echinoderms. Wiley Interdiscip Rev Dev Biol. 2018;7:e316.

29. Madeira F, Park YM, Lee J, Buso N, Gur T, Madhusoodanan N, et al. The EMBL-EBI search and sequence analysis tools APIs in 2019. Nucleic Acids Res. 2019;47: W636-41.

30. Kosakovsky Pond SL, Frost SDW. Not so different after all: a comparison of methods for detecting amino acid sites under selection. Mol Biol Evol. 2005;22:1208-22.

31. Murrell B, Moola S, Mabona A, Weighill T, Sheward D, Kosakovsky Pond SL, et al. FUBAR: a fast, unconstrained bayesian approximation for inferring selection. Mol Biol Evol. 2013;30:1196-205.

32. Swofford D PAUP*. Phylogenetic analysis using parsimony (*and Other Methods). Version 4.0b10 edn. 2002.

33. Hooper M, Hardy K, Handyside A, Hunter S, Monk M. HPRT-deficient (LeschNyhan) mouse embryos derived from germline colonization by cultured cells. Nature 1987;326:292-5.

34. Nagy A, Rossant J, Nagy R, Abramow-Newerly W, Roder JC. Derivation of completely cell culture-derived mice from early-passage embryonic stem cells. Proc Natl Acad Sci USA. 1993;90:8424-8.

35. Duyao MP, Auerbach AB, Ryan A, Persichetti F, Barnes GT, McNeil SM, et al. Inactivation of the mouse Huntington's disease gene homolog Hdh. Science 1995;269:407-10.

36. Rebuzzini P, Neri T, Mazzini G, Zuccotti M, Redi CA, Garagna S. Karyotype analysis of the euploid cell population of a mouse embryonic stem cell line revealed a high incidence of chromosome abnormalities that varied during culture. Cytogenet Genome Res. 2008;121:18-24.

37. Zheng $Q$, Cai X, Tan MH, Schaffert S, Arnold CP, Gong X, et al. Precise gene deletion and replacement using the CRISPR/Cas9 system in human cells. Biotechniques 2014;57:115-24.

38. Ewels $P$, Magnusson $M$, Lundin S, Käller M. MultiQC: summarize analysis results for multiple tools and samples in a single report. Bioinformatics 2016;32:3047-8.

39. Bolger AM, Lohse M, Usadel B. Trimmomatic: a flexible trimmer for Illumina sequence data. Bioinformatics 2014;30:2114-20.

40. Dobin A, Davis CA, Schlesinger F, Drenkow J, Zaleski C, Jha S, et al. STAR: ultrafast universal RNA-seq aligner. Bioinformatics 2013;29:15-21.

41. Love MI, Huber W, Anders S. Moderated estimation of fold change and dispersion for RNA-seq data with DESeq2. Genome Biol. 2014;15:550.

42. Alexa A, Rahnenführer J, Lengauer T. Improved scoring of functional groups from gene expression data by decorrelating GO graph structure. Bioinformatics 2006;22:1600-7.

43. Walter W, Sánchez-Cabo F, Ricote M. GOplot: an R package for visually combining expression data with functional analysis. Bioinformatics 2015;31:2912-4.

44. Bocchi VD, Conforti P, Vezzoli E, Besusso D, Cappadona C, Lischetti T, et al. The coding and long noncoding single-cell atlas of the developing human fetal striatum. Science 2021;372:eabf5759.

45. $\mathrm{Li} \mathrm{H}$, Liu J, Wu K, Chen Y. Insight into role of selection in the evolution of polyglutamine tracts in humans. PLoS One. 2012;7:e41167.

46. Schöls L, Bauer P, Schmidt T, Schulte T, Riess O. Autosomal dominant cerebellar ataxias: clinical features, genetics, and pathogenesis. Lancet Neurol. 2004;3:291-304.

47. Stoyas CA, La Spada AR. The CAG-polyglutamine repeat diseases: a clinical, molecular, genetic, and pathophysiologic nosology. Handb Clin Neurol. 2018;147:143-70.
48. Choudhry S, Mukerji M, Srivastava AK, Jain S, Brahmachari SK. CAG repeat instability at SCA2 locus: anchoring CAA interruptions and linked single nucleotide polymorphisms. Hum Mol Genet. 2001;10:2437-46.

49. Pouladi MA, Stanek LM, Xie Y, Franciosi S, Southwell AL, Deng Y, et al. Marked differences in neurochemistry and aggregates despite similar behavioural and neuropathological features of Huntington disease in the full-length BACHD and YAC128 mice. Hum Mol Genet. 2012;21:2219-32.

50. Pearson CE, Eichler EE, Lorenzetti D, Kramer SF, Zoghbi HY, Nelson DL, et al. Interruptions in the triplet repeats of SCA1 and FRAXA reduce the propensity and complexity of slipped strand DNA (S-DNA) formation. Biochemistry 1998:37:2701-8.

51. Hohjoh H, Akari H, Fujiwara Y, Tamura Y, Hirai H, Wada K. Molecular cloning and characterization of the common marmoset huntingtin gene. Gene 2009;432:60-6.

52. Tian $D$, Wang $Q$, Zhang $P$, Araki $H$, Yang S, Kreitman $M$, et al. Single-nucleotide mutation rate increases close to insertions/deletions in eukaryotes. Nature 2008;455:105-8.

53. Shimada MK, Sanbonmatsu R, Yamaguchi-Kabata $Y$, Yamasaki C, Suzuki $Y$, Chakraborty $\mathrm{R}$, et al. Selection pressure on human STR loci and its relevance in repeat expansion disease. Mol Genet Genom. 2016;291:1851-69.

54. White JK, Auerbach W, Duyao MP, Vonsattel JP, Gusella JF, Joyner AL, et al. Huntingtin is required for neurogenesis and is not impaired by the Huntington's disease CAG expansion. Nat Genet. 1997;17:404-10.

55. Barnes GT, Duyao MP, Ambrose CM, McNeil S, Persichetti F, Srinidhi J, et al. Mouse Huntington's disease gene homolog (Hdh). Somat Cell Mol Genet. 1994;20:87-97.

56. Lin B, Nasir J, MacDonald H, Hutchinson G, Graham RK, Rommens JM, et al. Sequence of the murine Huntington disease gene: evidence for conservation, alternate splicing and polymorphism in a triplet (CCG) repeat [corrected]. Hum Mol Genet. 1994;3:85-92.

57. Elkabetz Y, Panagiotakos G, Al Shamy G, Socci ND, Tabar V, Studer L. Human ES cell-derived neural rosettes reveal a functionally distinct early neural stem cell stage. Genes Dev. 2008;22:152-65.

58. Rigamonti D, Bauer JH, De-Fraja C, Conti L, Sipione S, Sciorati C, et al. Wild-type huntingtin protects from apoptosis upstream of caspase-3. J Neurosci. 2000;20:3705-13.

59. Krüger M, Schäfer K, Braun T. The homeobox containing gene Lbx1 is required for correct dorsal-ventral patterning of the neural tube. J Neurochem. 2002;82:774-82.

60. Lin H-H, Bell E, Uwanogho D, Perfect LW, Noristani H, Bates TJD, et al. Neuronatin promotes neural lineage in ESCs via $\mathrm{Ca}(2+)$ signaling. Stem Cells. 2010;28:1950-60.

61. Gaber ZB, Novitch BG. All the embryo's a stage, and Olig2 in its time plays many parts. Neuron 2011;69:833-5.

62. Rraklli V, Södersten E, Nyman U, Hagey DW, Holmberg J. Elevated levels of ZAC1 disrupt neurogenesis and promote rapid in vivo reprogramming. Stem Cell Res. 2016;16:1-9.

63. Toth $A B$, Shum $A K$, Prakriya M. Regulation of neurogenesis by calcium signaling. Cell Calcium. 2016;59:124-34.

64. Hř́bková H, Grabiec M, Klemová D, Slaninová I, Sun Y-M. Calcium signaling mediates five types of cell morphological changes to form neural rosettes. J Cell Sci. 2018;131:jcs206896.

65. Clabough EBD, Zeitlin SO. Deletion of the triplet repeat encoding polyglutamine within the mouse Huntington's disease gene results in subtle behavioral/motor phenotypes in vivo and elevated levels of ATP with cellular senescence in vitro. Hum Mol Genet. 2006;15:607-23.

66. Tosches MA. Developmental and genetic mechanisms of neural circuit evolution. Dev Biol. 2017:431:16-25.

67. Frenkel ZM, Trifonov EN. Origin and evolution of genes and genomes. Crucial role of triplet expansions. J Biomol Struct Dyn. 2012;30:201-10.

68. Hannan AJ. TRPing up the genome: Tandem repeat polymorphisms as dynamic sources of genetic variability in health and disease. Disco Med. 2010;10:314-21.

69. Orr HT, Zoghbi HY. Trinucleotide repeat disorders. Annu Rev Neurosci. 2007;30:575-621.

70. Li SH, Schilling G, Young WS, Li XJ, Margolis RL, Stine OC, et al. Huntington's disease gene (IT15) is widely expressed in human and rat tissues. Neuron 1993;11:985-93.

71. Strong TV, Tagle DA, Valdes JM, Elmer LW, Boehm K, Swaroop M, et al. Widespread expression of the human and rat Huntington's disease gene in brain and nonneural tissues. Nat Genet. 1993;5:259-65.

72. Yan J, Zhang H, Liu Y, Zhao F, Zhu S, Xie C, et al. Germline deletion of huntingtin causes male infertility and arrested spermiogenesis in mice. J Cell Sci. 2016;129:492-501.

73. Pearson CE, Nichol Edamura K, Cleary JD. Repeat instability: mechanisms of dynamic mutations. Nat Rev Genet. 2005;6:729-42. 
74. Genetic Modifiers of Huntington's Disease (GeM-HD) Consortium. Identification of genetic factors that modify clinical onset of Huntington's disease. Cell. 2015;162:516-26.

\section{ACKNOWLEDGEMENTS}

We thank all the suppliers from Academic Institution, Zoos and Bioparks that kindly provided us with tissues and genomic DNA. Specifically, Johannes Erritøze (House of Bird Research, Danmark) for providing bird tissue samples; Nicoletta Ancona (Acquario e Civica Stazione Idrobiologica Milano-Italy) for providing fish tissue samples (Labridae; Sparidae; Caproidae; Carangidae); Roberta Castiglioni (Parco Faunistico "Le Cornelle"-Italy) for providing body hairs of Callithrichinae (Saguinus oedipus); Silvia Colmegna (Istituto Zooprofilattico Sperimentale della Lombardia e dell'Emilia Romagna-Italy) for providing tissue samples from Galliformes (Gallus gallus); Enrico Merli (Ente forestale di Piacenza-Italy) for contributing to provide tissue samples from birds and mammals (Leporidae; Mustelidae); Maurizio Casiraghi (Department of Biotechnology and Biosciences, Università degli Studi di Milano Bicocca-Italy) and Adriano Martinoli, (Department of Theoretical and Applied Sciences, Università degli studi dell'Insubria-Italy) for providing tissue samples and genomic DNA from Chiropetera; Claudia Romeo (Department of Veterinary Sciences and Public Health, Università degli Studi di Milano-Italy) for providing mammal tissue samples (Sciuridae); Maristella Giurisato (Banca Tessuti Mammiferi Marini del Mediterraneo, Università di Padova-Italy) for providing samples of Delphinidae; Adriana Bellati (Department of Earth and Environmental Sciences, Università degli Studi di Pavia-Italy) for providing genomic DNA and tissues from reptiles (Phyllodactylidae) and birds; Riccardo Castiglia (Department of Biology and Biotechnology, Università di Roma La Sapienza-Italy) and Giovanni Amori (CNRInstitute of Ecosystem Study, Department of Biology and Biotechnology "C. Darwin" Università di Roma La Sapienza-Italy) for providing tissues from the reptile Podarcis siculus; Alessandro Bianchi and Irene Bartoletti (Istituto Zooprofilattico sperimentale della Lombardia e dell'Emilia Romagna-Italy) for providing tissues from Rupicapra rupicapra and for contributing to provide samples from birds (Accipitridae). We also thank Edoardo Razzetti (Museo Storia Naturale, Università degli Studi di Pavia-Italy) for contributing to provide mammalian and reptile specimens; Michela Podestà and Giorgio Bardelli (Milan Museum of Natural History_ltaly) for providing samples from the museum collection including Sciurus vulgaris, Rupicapra rupicapra, Stenella coeruleoalba, Phataginus tricuspis, Martes foina, Procavia capensis, Gerbillus gerbillus; Andrea Sforzi (Museo di Storia Naturale della Maremma-Italy) for providing spines from Hystrix cristata; Joanna Sumner (Museum Victoria, Melbourne, Australia) for spines from Tachyglossus aculeatus. We also thank Klaus Friedrich and Pilar Di Cerbo (Fondazione Bioparco di Roma, Italy) Valentina Truppa and Elisabetta Visalberghi (Istituto di Scienze e Tecnologie della Cognizione, CNR, Roma, Italy), Eva Martinez Nevado (Zoo, Madrid, Spain), Gabriel Alcantara de la Fuente (Faunia, Madrid, Spain), and Maria Teresa Abello (Parc Zoologic de Barcelona, Spain) for providing buccal swabs and blood samples from non-human primates. We thank Graziano Martello (Department of Molecular Medicine, University of Padova) for E14 cells and advices for their growth; Stefano Ricagno (Department of Biosciences, Università degli studi di Milano) for suggestions on HTT Q3PQ3 constructs design; Desiree Zambroni (Alembic, San Raffaele Hospital, Milano) and Chiara Cordiglieri (INGM Imaging facility) for their great support with imaging protocols and acquisition. This project was funded partially by Telethon Foundation GGP06250 and by CHDI Foundation, Inc. New York, (JSC A11103) a nonprofit biomedical research organization exclusively dedicated to developing therapeutics that will substantially improve the lives of HD-affected individuals. In particular, we wish to thank one donor who has been following and supporting our progress on this project for the past 10 years. A.C. is supported by the Portuguese National Funds through FCT with the Investigador FCT grant (IF/00209/2014).

\section{AUTHOR CONTRIBUTIONS}

RI, GF, CZ, MP, AC, APM and NS contributed to either sample collection and preparation and/or set up of $\mathrm{PCR}$ protocols, sequencing and data validation; CML and
OR contributed to collect tissue samples from non-human primates and their genomic DNA preparation; RI and GF contributed to preliminary bioinformatics analyses; RLR, TL, VDB, and DSH contributed to algorithm development and final bioinformatics analyses; EC and TFV. conceived the RMCE cell platform; RI, GF, CT and RZC contributed to the design of the experimental strategy to knock-out HTT in E14 $\mathrm{mES}$ cells; RI, CT and AS conducted cell biology experiments, genome editing of mES cells, functional and imaging studies of neural rosettes and neurons; GF contributed to the pseudogene discovery and initial analysis; RI and CZ to pseudogene quality control, standardization and validation; RI, VDB and DSH to final pseudogene bioinformatics analyses; RI, CT, RLR, CZ, TL, VDB, AS, GP and EC performed all RNAseq work, including strategy design, RNA-seq data analysis and interpretation; AS performed qPCR validation of RNA-seq data; RLR and CZ supervised analyses of PCR sequencing, raw data organization and data management for all sequences; $R I, C T, A S$ were responsible for preparing final supplementary data S18-S20 and S23-S26; GF, CZ for data S1, S19, S22; RLR for data S1-S17; TL, VDB for data S18, S21; RI, GF, CT, RLR $C Z, T L, V D B, D S H$, and EC wrote the paper which was read, commented and reviewed by all authors. EC coordinated the study, administered the experiments, secured the funding and ensured that the descriptions contained in the final version of the paper are accurate and agreed by all authors. EC carries the responsibility for non-scientific disclosure of the data due to sensitive information for HD patients. The contributions in each section of this paragraph reflect the order of the authors.

\section{COMPETING INTERESTS}

The authors declare no competing interests.

\section{ETHICS APPROVAL}

The animal samples were collected in according to the ethical rules of different centers. The use of DNA samples was approved by O.P.B.A. (OPBA_84_2018) of the University of Milan.

\section{ADDITIONAL INFORMATION}

Supplementary information The online version contains supplementary material available at https://doi.org/10.1038/s41418-021-00914-9.

Correspondence and requests for materials should be addressed to Elena Cattaneo.

Reprints and permission information is available at http://www.nature.com/ reprints

Publisher's note Springer Nature remains neutral with regard to jurisdictional claims in published maps and institutional affiliations.

\begin{abstract}
Open Access This article is licensed under a Creative Commons Attribution 4.0 International License, which permits use, sharing, adaptation, distribution and reproduction in any medium or format, as long as you give appropriate credit to the original author(s) and the source, provide a link to the Creative Commons license, and indicate if changes were made. The images or other third party material in this article are included in the article's Creative Commons license, unless indicated otherwise in a credit line to the material. If material is not included in the article's Creative Commons license and your intended use is not permitted by statutory regulation or exceeds the permitted use, you will need to obtain permission directly from the copyright holder. To view a copy of this license, visit http://creativecommons. org/licenses/by/4.0/.
\end{abstract}

(c) The Author(s) 2021 